\title{
Guidance by followers ensures long-range coordination of cell migration through $\alpha$-Catenin mechanoperception
}

Arthur Boutillon ${ }^{1}$, Diego Jahn², Sebastián González-Tirado², Jörn Starruß², Lutz Brusch², Nicolas B. David $^{1}$

${ }^{1}$ Laboratory for Optics and Biosciences, CNRS UMR7645, INSERM U1182, Institut Polytechnique de Paris, 91128 Palaiseau, France.

${ }^{2}$ Center for Information Services and High Performance Computing, Technische Universität Dresden, Dresden, Germany.

\begin{abstract}
Morphogenesis, wound healing and some cancer metastases depend upon migration of cell collectives that need to be guided to their destination as well as coordinated with other cell movements. During zebrafish gastrulation, extension of the embryonic axis is led by the mesendodermal polster that migrates towards the animal pole, followed by axial mesoderm that is undergoing convergence and extension. We here investigate how polster cells are guided towards the animal pole. Using a combination of precise laser ablations, advanced transplantations and functional as well as silico approaches, we establish that the directional information guiding polster cells is mechanical, and is provided by the anteriorward migration of the following cells. This information is detected by cell-cell contact through E-Cadherin/ $\alpha$-Catenin mechanotransduction and propagates from cell to cell over the whole tissue. Such guidance of migrating cells by followers ensures long-range coordination of movements and developmental robustness.
\end{abstract}




\section{Introduction}

Cell migration executes and orchestrates key events in development, homeostasis, and disease (Yamada and Sixt, 2019). Apart from a few examples of cells spreading through random migrations (Borrell and Marín, 2006; Pézeron et al., 2008), most cell migration events are precisely guided in vivo, with chemical or physical environmental cues orienting cell movement (Shellard and Mayor, 2020). The past decade has highlighted that many cells do not undertake migration on their own, but are rather influenced by neighbouring cells, in so-called collective migrations (Norden and Lecaudey, 2019; Scarpa and Mayor, 2016; Schumacher, 2019). This is relevant not only to epithelial cells that need to migrate while maintaining close contacts with their neighbours, but also to some mesenchymal cells. Though not bound to their neighbours, mesenchymal cells rely on interactions with surrounding cells to properly migrate. The best characterized instance is neural crest cells in Xenopus. In this system, cells undergo contact inhibition of locomotion (CIL), whereby contacting cells repolarize away from one another (Carmona-Fontaine et al., 2008; Scarpa et al., 2015). This provides outward polarity for cells in clusters and allows an efficient response to a chemoattractant. Concomitantly, group cohesion is provided by co-attraction, whereby neural crest cells express a chemoattractant to which they themselves respond (Carmona-Fontaine et al., 2011).

In addition to being directionally guided, many cell migrations need to be tightly coordinated with other cell movements. This is particularly true during development when many concomitant cell movements shape the forming embryo. Recent work has proposed mechanical interactions as a way to couple movements of different cell populations (Das et al., 2019; Xiong et al., 2020). However, the cellular bases for such interactions, and how long-range coordination can be achieved, remains poorly explored owing to the challenge of both properly imaging and quantifying cell migration in vivo, and of physically altering the cell's environment to probe the origin and the nature of guidance cues.

Here, we investigate these questions in zebrafish, analysing how the migration of the anterior axial mesendoderm is directed towards the animal pole. At the onset of gastrulation, the first cells to internalize on the dorsal side of the embryo are precursors of the polster (hereafter referred to as polster cells) (Kimmel et al., 1995; Solnica-Krezel et al., 1995). From the embryonic organiser, they migrate in a straight line towards the animal pole, leading the extension of the axis, and are followed by more posterior axial mesodermal cells, including posterior prechordal plate precursors and notochord precursors (Montero et al., 2005; Figure S1A). All these cells express the Tg(gsc:GFP) transgene, which also labels some endodermal cells (Figure S1A, Barone et al., 2017). Although different pathways have been implicated in the migration of polster cells (Blanco et al., 
2007; Kai et al., 2008; Montero et al., 2003, 2005; Shimizu et al., 2005; Yamashita et al., 2002, 2004), how polster cells are guided towards the animal pole remains unknown. In particular, loss-offunction of Wnt/PCP pathway components affects their migration directionality (Heisenberg et al., 2000; Ulrich et al., 2005), suggesting a potential instructive role of the Wnt/PCP pathway in cell guidance. However, ubiquitous optogenetic activation recently demonstrated unambiguously that the Wnt/PCP pathway plays only a permissive role in these cells (Čapek et al., 2019), reopening the question of how they are guided. A few years ago, we demonstrated that migration of polster cells is a collective process; cells require E-cadherin dependent contacts with their neighbours to perceive directional information and extend protrusions towards the animal pole (Dumortier et al., 2012). Yet the nature of the directional information transmitted at cell contacts and its origin have remained unknown.

In this study, we used complementary approaches, including precise 3D laser ablations and cell transplants, to map the directional information that guides polster cell migration. We identify follower cells as a source: it is their anteriorward movement that provides propagating mechanical information and orients the migration of polster cells. Cell autonomous inhibition of motility further revealed that mechanical information propagates from cell to cell, through their active migration. Looking for the molecular pathway enabling mechanosensation, we find that the mechanosensitive domain of $\alpha$-Catenin is required for cell orientation. These results lead to a model of axis extension in which the anteriormost structure, the polster, is guided by more posterior, follower, cells. This mechanical 'guidance by followers' accounts for the long-range coordination of movements of the different cell populations forming the axis, and provides a basis for its developmental robustness.

\section{Results}

\section{Polster cells do not exhibit Contact Inhibition of Locomotion nor co-attraction}

Though they form a group, polster cells appear mesenchymal: all cells form protrusions (Dumortier et al., 2012) and gaps exist between cells (Smutny et al., 2017). Investigating the mechanisms ensuring their collective migration, we first sought to test if polster cells rely on similar processes as those driving neural crest cell migration, namely Contact Inhibition of Locomotion (CIL) and co-attraction.

We first tested CIL in polster cells by performing mixing assays (Carmona-Fontaine et al., 2008). Two groups of differently-labelled polster cells were transplanted next to each other at the animal pole of an early gastrula embryo, a region devoid of hypoblast cells that could otherwise 
interfere with polster cells migration (Figure 1A, Movie S1). Interpenetration of the two groups was measured after 90 minutes as the normalized area of overlap between the two cell populations. The observed broad interpenetration argues against the existence of CIL, as illustrated by simulating the assay with cells displaying CIL or not (see Methods). Second, if CIL was occurring, cells would be expected to change direction upon contact with others (Carmona-Fontaine et al., 2008). We transplanted polster cells expressing H2B-mCherry at the animal pole of a host embryo, tracked their movements, and measured their change in direction upon collision with another polster cell (Figure 1B, Movie S1). As controls, we compared these changes to the natural tendency of these cells to change direction on their own, in absence of collision. We observed no difference in changes of direction with or without collision, arguing against a CIL behaviour.

To assess co-attraction, we transplanted single polster cells expressing Lifeact-mCherry in front of the polster and quantified their movement and protrusion orientations (Figure 1C, Movie S1). While isolated, cells displayed no clear preferred direction, and an almost random orientation of protrusions, suggesting the absence of strong co-attraction. We nevertheless noticed a small bias of movements and protrusions of isolated cells towards the polster, which could arise from a weak coattraction or from the friction forces exerted by the ectoderm (Smutny et al., 2017). To discriminate between these possibilities, we transplanted two groups of differently-labelled polster cells at the animal pole, separated by some distance, and measured whether they attracted each other (Figure 1D, Movie S1) (Carmona-Fontaine et al., 2011). After $90 \mathrm{~min}$, the distance between centroids had increased by $47 \pm 28 \mu \mathrm{m}$, arguing against any co-attraction. Furthermore, we observed that groups of cells at the animal pole spread as small clusters or isolated cells; they did not remain compact as would be expected in the case of co-attraction. We thus could not detect signs of CIL nor coattraction in polster cells.

\section{The directional information guiding the polster is not within the polster.}

We therefore looked for other mechanisms that could guide polster cell collective migration. In previous work, we observed that isolated polster cells lack orientation cues, which are restored upon contact with a migrating polster, suggesting that directional cues are transmitted through cellcell contacts (Dumortier et al., 2012). We sought to map the origin of this directional information. To do so, we developed large 3D ablations, to sever the polster at different positions and identify which regions are required for its oriented migration and where the directional information might come from. The use of non-linear optics provided sufficient axial resolution not to affect the yolk cell underneath, nor the ectoderm above (Figure S1B). Treated embryos could develop until at least 24 hours post fertilization (hpf) and presented only a slight delay compared to controls, suggesting that the laser treatment was not harmful (Figure S1C). 
We first tested if the first row of polster cells acts as leaders to guide follower cells, a mechanism described in many instances of collective migration (Haeger et al., 2015; Vishwakarma et al., 2020). The front row of cells was ablated and the movement of posterior cells was quantified by tracking their H2B-mCherry-labelled nuclei (Movies S2 and S3). For each cell, we measured both instantaneous speed (Figure S2B), referred to as absolute speed, and its axial component, in the direction of the animal pole, referred to as axial speed (Figure 2B). Removal of front cells did not affect follower polster cells' absolute (Figure S2B) nor axial speed (Figure 2B). To confirm that front cells do not guide follower cells, we directly quantified cell protrusion orientation before and after ablation by transplanting a few polster cells expressing Lifeact-mCherry into the polster of an unlabelled host (Figure 2A). Providing scarce labelling, this allows precise quantification of cell protrusions and measurement of their orientation (Boutillon et al., 2018). Ablation of the front cell row had no significant effect on protrusion orientation of follower cells (Figure $2 \mathrm{C}$ ), confirming that front cells are not required to guide them.

To identify the source of directional information, ablations were performed at different antero-posterior positions. First, to isolate the anterior half from the posterior part, middle polster cells were removed by a transversal ablation (Figure 2A, Movie S3). While the absolute speed of anterior cells was not affected by isolation (Figure S2B), their axial speed (animalward motion) decreased dramatically (Figure 2B). As a control for non-specific effects induced by laser ablation, we performed sagittal ablations, parallel to the direction of migration, separating the left and right anterior polster, but leaving each side in contact with the posterior polster (Figure 2A, Movie S3). Such an ablation did not significantly reduce axial speed (Figure 2B). Decrease of axial speed but not of absolute speed upon separation of the entire anterior half of the polster suggested that cells exhibited poorer orientation. We directly tested this by quantifying protrusion orientation of LifeactmCherry expressing cells transplanted in the polster before laser ablations. Whereas control sagittal ablations did not affect cell orientation, transversal ablations strongly disrupted protrusion orientation of cells in the isolated anterior polster (Figure 2C). Interestingly, cells in the posterior half were still oriented (Figure $2 \mathrm{C}$ ) and their axial speed was higher than cells in the anterior part (Figure $\mathrm{S} 2 \mathrm{C}$ ) suggesting that the directional information guiding the migration is present in the posterior polster.

To test if the directional information is contained in the posterior polster, we performed similar ablation experiments, this time separating the entire polster from the following axial mesoderm (Figures 2A and S1D, Movie S3). Strikingly, this procedure abolished the animalward movement of polster cells, without affecting their absolute speed (Figures 2B and S2B). Consistent with this loss of direction, the orientation of protrusions was completely lost after ablation (Figure 2C). These experiments reveal that, contrary to what we previously proposed (Dumortier et al., 
2012), the directional information orienting polster cells is not contained within the polster itself, but rather seems to be provided by contact with the posterior axial mesoderm.

\section{Contact with the following axial mesoderm is required for polster oriented migration}

To directly test if the following axial mesoderm is the source of directional information, we performed more posterior laser ablations, leaving some axial mesoderm in contact with the polster ( $3.7 \pm 1$ rows of cells) (Figures $2 \mathrm{~A}$ and S1D, Movie S3). This largely restored polster migration compared to ablations separating the polster from the following mesoderm (Figure 2B). Consistent with this, cell orientation was also restored (Figure $2 \mathrm{C}$ ) suggesting that contact between polster and axial mesoderm is necessary for proper orientation and migration of polster cells. This idea is further supported by the observation that, in ablations separating the polster from the following axial mesoderm, the axial mesoderm continued elongating, resulting in wound closure in $24 \pm 2 \min$ ( $n=14$ embryos). A few minutes after wound closure, polster migration resumed (Figure S2D) leading to normal development at $24 \mathrm{hpf}$ (Figure S1C).

We sought to confirm these surprising results with a second, independent approach. Using large glass pipettes, we removed the endogenous polster from $\mathrm{Tg}$ (gsc:GFP) embryos (Figure 3A). In such embryos, axial mesoderm continues elongating. We then transplanted a group of polster cells, with H2B-mCherry labelled nuclei, ahead of the axial mesoderm (Figure 3B, Movie S4). While isolated, these cells spread isotropically, migrating without any preferred direction. In contrast, after contact with the axial mesoderm, polster cells migrated towards the animal pole (Figures $3 \mathrm{C}$ and 3D). Repeating this experiment with some Lifeact-mCherry-labelled cells among the transplanted polster cells, we observed that cell protrusion orientation was randomly distributed before contact but became oriented towards the animal pole once the transplanted group was contacted by the axial mesoderm (Figure 3E). In accordance with laser ablations, these observations demonstrate that the polster requires contact with the following axial mesoderm to orient its migration towards the animal pole.

\section{Extension of the following axial mesoderm is required for polster migration orientation}

Trying to identify the nature of the directional information, we wondered if polster cells simply required contact with the axial mesoderm, or if they required the animalward movement of the axis undergoing convergence-extension (Myers et al., 2002). To address this question, we examined the migration of a wild-type polster in front of a defective axis: we genetically slowed axis extension in embryos (see below) and replaced their polster with wild-type polster cells (Figure 4A). As a control, we performed polster replacements between wild-type embryos, and observed no 
difference in speed of the polster or of the axial mesoderm compared to untreated embryos (Figure 4A). Inhibiting the non-canonical Wnt-PCP pathway affects axis extension (Čapek et al., 2019; Heisenberg et al., 2000; Ulrich et al., 2005). Consistently, expression of Dsh-DEP+, a dominant negative form of Dsh specifically blocking the PCP pathway (Tada and Smith, 2000), strongly slowed axial mesoderm extension (Figure 4A). Importantly, in Dsh-DEP+ expressing embryos, a transplanted, wild-type polster showed a similar reduction in speed (Figure 4A). A second genetic manipulation that dramatically slows down axial mesoderm extension is expression of Rac1 N17 (Figure 4A), a dominant negative form of the Rac1 small GTPase (DN-Rac1) (Tahinci and Symes, 2003). In DN-Rac1expressing embryos, the animalward movement of a transplanted, wild-type polster was essentially abrogated (Figure 4A). These results demonstrate that the extension of axial mesoderm, not simply contact with it, is required for the migration of the polster.

\section{Active migration of polster cells is required for axis elongation}

That the axial mesoderm extends without polster cells, and that this extension is required for polster migration raised the possibility that the polster is simply passively pushed towards the animal pole by the independently-extending axis. To test whether active migration of polster cells is required, we used DN-Rac1 to inhibit their migration (Dumortier et al., 2012), and then transplanted a non-migrating polster into a wild-type embryo. A DN-Rac1-expressing polster replacing that of a wild-type embryo did not move towards the animal pole (Figure $4 \mathrm{~B}$ ) and blocked the elongation of the axial mesoderm. Active migration of polster cells is thus required for their movement towards the animal pole. Interestingly, however, we noticed that when replacing the polster by a small number of DN-Rac1 cells, these cells, while non-motile, were efficiently displaced towards the animal pole by the extending axis (Figures $4 \mathrm{~B}$ and $4 \mathrm{C}$ ), demonstrating that extension of the axis does generate pushing forces, though not sufficient to displace an entire polster.

\section{Polster cells can be oriented by another migrating tissue}

That axial cells exert pushing forces and that their animalward movement is required to orient polster cell migration suggest that the movement of the axial mesoderm provides mechanical information that orients the active migration of polster cells. We could not, however, rule out that axial cells provide a chemical cue transmitted only through cell-cell contacts. In this case, axis extension would be required to maintain contact and hence transmission of the chemical cue between the two tissues. To distinguish between mechanical and chemical signal, we first transplanted wild-type cells into a polster expressing DN-Rac1 (Figure 4C). Such a polster remains in contact with the axis, so any chemical signal should propagate normally. In such a context, wild-type cells formed protrusions at the same frequency as in a wild-type replaced polster (Figure $4 \mathrm{C}$ inlay). 
Protrusion orientation, however, was randomized (Figure 4C). This result argues against a chemical signal transmitted from cell to cell.

If polster cells become oriented by the movement of more posterior cells, they may not specifically require interactions with axial mesoderm, but could be oriented by other migrating cells. To test this, we transplanted polster cells ahead of the lateral mesoderm (Figure 5A, Movie S5). Lateral mesoderm cells, visible in the $T g(t b \times 16: G F P)$ line (Figure 5A), internalize at the embryonic margin before migrating towards the animal pole (Solnica-Krezel et al., 1995). We quantified polster cell migration and orientation before and after contact with the lateral mesoderm. Before contact, polster cells moved and extended protrusions without a preferred orientation. Upon contact with the lateral mesoderm, they aligned both their migration and protrusions (Figures 5B and 5D) with the movement of the lateral mesoderm. This experiment shows that guidance of polster migration is not specific to posterior axial mesoderm, but can be triggered by another migrating tissue.

\section{Polster cells are oriented by actively migrating neighbours}

A mechanical signal orienting migration could arise from polster cells being pushed and displaced or from these cells sensing the active migration of their direct neighbours. To distinguish between these sources, we transplanted a few wild-type cells within a small group of DN-Rac1 expressing cells at the front of the polster. Transplanted cells are thus still displaced by the extension of the axis (the small DN-Rac1 cell cluster is displaced, see Figure 4B) but are only in contact with non-motile DN-Rac1 cells. In this context, the protrusions of wild-type cells without actively migrating neighbours were less oriented than those of wild-type cells in a similar small group of other wild-type cells (Figure 4D): polster cells thus require contact with motile neighbours to become oriented. This suggests that motile cells exert forces on their neighbours that serve as directional information.

Adherens junctions ensure cell-cell adhesion and can elicit mechanotransduction, and are therefore strong candidates to transmit and perceive forces. If true, cadherin should be required both within polster cells to perceive forces, and in neighbouring cells, to apply forces. We previously demonstrated, and here confirmed, that E-cadherin knock-down cell-autonomously leads to a loss of protrusion orientation (Dumortier et al., 2012), which is rescued by expression of E-cadherin-GFP (Figure S3A). We tested for an E-cadherin requirement in neighbouring cells by transplanting wildtype cells within a small E-cadherin knocked-down cell cluster. The absence of E-cadherin in neighbours led to a loss of orientation of wild-type cells (Figure 4D). Cadherins are thus required both within polster cells and in their migrating neighbours to orient cell protrusions, suggesting that cadherins transmit forces and their inherent directional information.

\section{Polster cell orientation requires $\alpha$-Catenin and Vinculin mediated mechanosensation}


In some cells, however, Cadherins can influence cell migration without their cytoplasmic domain, in an adhesion and force independent process (Nguyen and Mège, 2016). We thus checked whether the cytoplasmic domain of E-cadherin is required for cell orientation. E-cadherin knockeddown cells, expressing a form of E-cadherin lacking the intracellular domain (E-cadherin $\Delta$ cyto, Maître et al., 2012), were transplanted into a wild-type polster. Contrary to wild-type E-cadherin, expression of E-cadherin $\Delta$ cyto did not rescue protrusion orientation (Figure S3A), consistent with a mechanotransducer role of E-cadherin.

Looking for downstream effectors of E-cadherin, Plakoglobin was an obvious candidate. In Xenopus polster cells (anterior axial mesendoderm), it is recruited to adherens junctions upon application of tension and is required for cell orientation in response to tension (Sonavane et al., 2017; Weber et al., 2012). In zebrafish, there are two paralogues of plakoglobin (jupa \& jupb). Knocking down both with morpholinos led to cardiac oedema and embryonic death at 1 and $3 \mathrm{dpf}$, as previously described (Figure S3C) (Martin et al., 2009). However, the double knock-down did not affect protrusion orientation of polster cells (Figure S3B).

Another component of adherens junctions, $\alpha$-Catenin, links E-cadherin to actin and can ensure mechanotransduction: under tension $\alpha$-Catenin undergoes a conformational change, revealing the MI binding site for Vinculin and other proteins (Kobielak et al., 2004; Nieset et al., 1997; Pokutta et al., 2002; Yonemura et al., 2010). We thus tested whether $\alpha$-Catenin mechanosensation is required for polster cell orientation. Knock-down of $\alpha$-Catenin reduced protrusion orientation, which could be rescued by co-injection of $\alpha$-Catenin mRNA, indicating that $\alpha$-Catenin is required for polster cell orientation (Figure 6A, Movie S6). To determine if $\alpha$-Catenin is required as a link between Ecadherin and the cytoskeleton or as a mechanosensor, we tried rescuing the knock-down with the $\alpha$ Catenin $\triangle$ VBS construct, which still binds actin but lacks mechanosensation (Han et al., 2016; Huveneers et al., 2012; Twiss et al., 2012). As expected, this $\alpha$-Catenin $\Delta V B S$ partially rescued developmental defects induced by $\alpha$-Catenin knock-down (Han et al., 2016) (Figure S3D). Yet, it did not restore polster cell orientation (Figure 6B) suggesting that the mechanosensory function of $\alpha$ Catenin is required to orient polster cells. To confirm this, we performed similar rescue experiments with the L344P form of $\alpha$-Catenin, which bears a point mutation in the Vinculin binding site, preventing the tension-dependent recruitment of Vinculin (Seddiki et al., 2018). This tensioninsensitive form did not rescue cell orientation (Figures 6B and S3D). As the Vinculin binding domain of $\alpha$-Catenin appeared to be required for cell orientation, we directly tested the involvement of Vinculin, knocking down the two zebrafish paralogues (Figure 6A, Movie S6). Consistent with the $\alpha$ Catenin results, Vinculin is required for proper cell orientation (Figure 6C). These results establish that polster cell orientation is driven by mechanotransduction mediated through E-cadherin, $\alpha$ Catenin and Vinculin. 


\section{In silico simulations reveal the emergence of a robust collective behaviour}

Overall, our results suggest that each polster cell is oriented by stresses exerted by actively migrating neighbours. To address which statiscal properties can emerge from such cell interactions and if this is sufficient to account for the observed collective migration of polster cells, we turned to an in silico approach and used the Cellular Potts Model (Graner and Glazier, 1992) in the modelling and simulation framework Morpheus (Starruß et al., 2014). Briefly, polster cells were given a Run and Tumble behaviour (see Methods and Figure S4) and a tendency to align with cells pulling on them (migrating towards them). Such a rule proved sufficient to induce the collective migration of polster cells, followed by animalward migrating axial mesodermal cells (Figure 7A, Movie S7). It also correctly reproduced experimental observations upon laser ablations (Movie S7). In particular, we could notice in simulations a slight backward movement of the cells at the posterior edge of the isolated group, and a tendency of the most anterior cells to migrate out of the group after ablations, two characteristics we could then identify in experimental data (Figure S5A). Of note, other simple rules, like correlating the movement of one cell to the movement of any of its neighbours, as observed in confluent epithelia (Poujade et al., 2007) for instance, could not reproduce experimental results (Movie S7).

We also mimicked experiments in which the speed of the axial mesoderm is reduced (presented in Figure 4A). Consistent with experimental observations, the speed of the simulated polster diminished, even though individual polster cell properties were unchanged (Figure 7B, Movie S7). This reduction of group speed stems from an emergent property of the interacting multicellular system: in simulations, we measured the orientation coherence of polster cells and found it to be linearly dependent on the speed of posterior cells (Figure S5B), so that when axis speed is reduced, polster cells maintain their individual speed, but are less oriented, leading to a reduction of the group speed. We directly tested this model prediction by measuring cell movement orientation in the experiments where axis extension was slowed and found the same striking correlation between axis speed and polster cell orientation (Figure S5B). Guidance by followers thus ensures axis integrity as an emergent property from cell-cell interactions.

\section{Discussion}


Precise guidance of migrating cells is required to achieve proper development and morphogenesis. In vitro, many chemical and physical cues can orient cell migration, but it is not clear how such cues can guide cells over long distances in the dynamic environment of the developing embryo. Here, focusing on the forming embryonic axis, we have shown that the polster, the anteriormost cell group, rather than being attracted to its destination by long-range signals, is guided by mechanical cues provided by the anteriorward movement of more posterior cells, creating a robust and self-organizing system. Interestingly, the idea that a mechanical information can propagate and coordinate movements of cells at a distance was recently proposed in two other systems (Das et al., 2019; Xiong et al., 2020). Yet, what generates the mechanical signal, how the mechanical information is perceived, and what cellular properties these signals regulate remained unknown. Here, we established that the mechanical signal is generated by the active migration of follower cells, that it is perceived through the E-cadherin - $\alpha$-Catenin - Vinculin pathway, and that it controls cell orientation.

In vitro work in Xenopus established that migration of polster cells can be guided by pulling forces applied at the cell rear, leading to a model of collective migration in which more posterior cells would orient migration by acting as a drag (Behrndt and Heisenberg, 2012; Weber et al., 2012). Our observations that posterior cells can migrate without polster cells (Figure 3), that they can exert anteriorward forces sufficient to displace a group of non-motile cells (Figure 4B), and that orientation of polster cells requires the anteriorward movement of posterior cells (Figure 4A) demonstrate that, in zebrafish, posterior cells are not acting as a drag, but that it is their active migration which orients polster migration. How this active migration is perceived is, however, not obvious. One possibility is that it generates compressive forces perceived by polster cells. However, our finding that $\alpha$-Catenin mechanosensing is required to orient cells rather points to cells perceiving tensile forces, as $\alpha$ Catenin is opened under tension (Hoffman and Yap, 2015). Compression could then be detected as a source of shear stress: passive pushing of a cell attached to its neighbours would put adherens junctions under tension. Such friction forces have been identified between polster cells and the overlying ectoderm (Smutny et al., 2017), and shear stress induced by neighbours was recently implicated in the collective migration of endothelial cells (Patel et al., 2020). However, our observation that cells require their direct neighbours to be actively migrating and to express $\mathrm{E}$ cadherin instead point to a third possibility. To migrate, cells emit anteriorward protrusions on which they pull to progress. These protrusions thus exert tensile forces on anterior neighbours, which could be used to orient them (Figure 7C), ensuring cell guidance by followers. In this case, protrusions would serve not only as grapples used to move forward (Lauffenburger and Horwitz, 1996) but also as a means to transmit directional information, a process reminiscent of Drosophila border cells 
(Mishra et al., 2019). Simulations of such cell-cell interactions faithfully reproduced experimental observations, demonstrating that this mechanism is sufficient to account for the collective behaviour of polster cells.

\section{How are cells oriented by their neighbours?}

Our model suggests that cells perceive stresses exerted at their surface by neighbours and use them to orient actin rich protrusions. We identified the E-cadherin, $\alpha$-Catenin, Vinculin pathway as the involved mechanosensor, in line with previous reports showing that stretch induces an $\alpha$ Catenin change of conformation and Vinculin recruitment (Hoffman and Yap, 2015; Kim et al., 2015; Ladoux et al., 2015). In epithelial cells, this leads to reinforcement of adherens junctions (Jurado et al., 2016) and has been involved in controlling collective epithelial behaviours (Bazellières et al., 2015; Seddiki et al., 2018). How E-cadherin, $\alpha$-Catenin and Vinculin control orientation of actin rich protrusions remains to be identified. One candidate is Merlin, as in collectively migrating epithelia, it transduces mechanosensation to coordinate Rac1 activity and lamellipodium formation (Das et al., 2015). Apart from Merlin, very little is known on how adherens junctions regulate cytoskeleton dynamics and cell migration (Vishwakarma et al., 2020) Nevertheless, there is accumulating evidence that mechanosensation at cell-cell contacts has a key role in coordinating many cell behaviours (Hirata et al., 2020; Vassilev et al., 2017; Vishwakarma et al., 2018). Unravelling the events downstream of $\alpha$-Catenin and Vinculin is thus a very exciting avenue for future work, and the zebrafish polster appears as a convenient model system to progress on this line of inquiry.

\section{What is driving axis elongation?}

We demonstrated that orientation of polster cells, the cells that lead the formation of the embryonic axis, depends on the anteriorward movement of more posterior, following, cells. A question remains however: what is driving the movement of following cells? It was recently established that a group of cells, located just posterior to the polster, expresses $p c d h 18 a$, which, by modulating E-cadherin recycling, would transform them into a fast-migrating group (Bosze et al., 2020) which represents an attractive candidate to guide polster migration. If true, this, however, only shifts the question to how these cells are oriented towards the animal pole. One likely hypothesis is that these cells use the very same mechanism as the one we unveiled for the most anterior ones, relying on the active migration of following cells to orient their movement. In such an hypothesis, the system would be fuelled during early gastrulation by the continuous internalisation of cells at the margin (Giger and David, 2017; Krens et al., 2017) which serves as the symmetry breaking event. This 
would provide a very simple, yet very robust, coupling of two key gastrulation movements, mesoderm internalization and axis elongation.

\section{Coordination of movements}

During development, and during gastrulation in particular, many different cell movements are taking place concomitantly. How the different cell populations coordinate their movements to ensure proper morphogenesis remains largely unknown. For axis elongation in fish, it is noticeable that mutants affecting extension of notochord precursors also affect progression of the anterior polster cells (Heisenberg et al., 2000; Topczewski et al., 2001). This co-occurrence could arise from these cells using similar pathways for their migration. Indeed, the Wnt/PCP pathway appears to be required both for the mediolateral intercalations driving posterior axis extension and for the directed migration of polster cells (Roszko et al., 2009). Alternatively, there may be mechanisms ensuring coordination of the two movements. Using transplants of entire polsters, we observed that progression of a wild-type polster is delayed when notochord progression is genetically delayed, revealing the existence of coordination mechanisms ensuring the integrity of the embryonic axis.

Achieving such coordination is not trivial. As illustrated by in silico simulations, systems in which cells have a directed motion towards the animal pole are very sensitive to any difference in speed between polster and more posterior cells (Figure 7B). Adjusting polster speed to axial speed would imply that axial cells instruct polster cells to slow down. On the opposite, guidance by followers spontaneously provides robustness to the system. The mechanical information that propagates through the tissue modulates cell orientation, and this is sufficient to modulate speed of the entire group. Guidance by followers, in which the cell-to-cell propagation of mechanical information orients cell polarity, is thus a very simple, yet very effective way of ensuring long-range coordination of cell movements and self-organized guidance. Such mechanical coordination is likely to control morphogenesis in other contexts, in embryonic development, organogenesis or cancer cell migration.

\section{Acknowledgements}

We thank S. Escot and F. Rosa for critically reading the manuscript; F. Graner and W. de Back for initial help with simulations, C. Wyart, J. de Rooij, E. Raz, R.M. Mège for fish lines and plasmids; Emilie Menant for fish care. We thank Pierre Mahou and the Polytechnique Bioimaging Facility for 
assistance with live imaging on their equipment partly supported by Région lle-de-France (interDIM) and Agence Nationale de la Recherche (ANR-11-EQPX-0029 Morphoscope2, ANR-10-INBS-04 France Biolmaging). This work was supported by the ANR grants 15-CE13-0016-1, 18-CE13-0024, 20-CE130016. A.B. was supported by the Ministère de l'Enseignement Supérieur et de la Recherche, N.B.D. was supported by the Centre National de la Recherche Scientifique. L.B. acknowledges support by the BMBF through FitMultiCell grant 031L0159B. Model simulations for Bayesian parameter inference were performed on HPC resources granted by the ZIH at TU Dresden.

\section{Author Contributions}

$A B$ and ND conceived experiments, which were performed by AB. DJ, SGT, JS, LB and ND developed the simulation model, performed the parameter estimation, ran simulations and wrote the corresponding methods section. $A B$ and ND analysed data, wrote the manuscript. $L B$ and ND secured funding.

\section{Declaration of Interests}

The authors declare no competing interests.

\section{Figure Legends}

Figure 1: Polster cells do not exhibit CIL nor CoA. (A) Group mixing assay: transplantation of two adjacent, differently labelled groups of polster cells (red and green). Simulated cells display migration characteristics similar to polster cells (see also Figure S4), with or without CIL behaviour. Overlap, highlighted in yellow, is measured at $90 \mathrm{~min}$ for experimental ( $n=11$ embryos) and simulated data. (B) Collisions of polster cells transplanted at the animal pole. Change in direction $(\theta)$ is measured as the angle between the displacement vector before (red arrow) and after (other colours) a given time step, upon collision or not. Cumulative frequency of angle $\theta$ is displayed. Red: change in direction upon collision ( $n=82$ cells); gray: 100 bootstrapped datasets of change in direction in absence of collision; black: combination of all 100 bootstrapped datasets. (C) Unique cells transplanted $58 \pm 25$ $\mu \mathrm{m}$ ahead of the polster ( $n=19$ cells). Cell trajectories and orientation of actin-rich protrusions (angle between the direction of the protrusion and the direction of the animal pole) are displayed before and after contact with the polster. Asterisks mark the initial position of the transplanted cell; white arrow indicates direction of the Animal Pole (AP) (D) Group attraction assay: transplantation of two 
differently labelled groups of polster cells ( $n=7$ embryos), initially $166 \pm 38 \mu \mathrm{m}$ apart. White crosses mark group centroids; dashed lines mark the distance between centroids, which is plotted at 0 and $90 \mathrm{~min}$. In this and following figures, ns: p.value $\geq 0.05 ;^{*}: p<0.05{ }^{* *}: p<0.01 ; * * * p<10^{-3} ; * * * * p<10^{-}$ 4.

Figure 2: Directional information is not contained in the polster. (A) Laser ablations at varying anteroposterior positions and/or orientations. Representative images of ablations, taken from experiments in which a few Lifeact-mCherry expressing cells were transplanted in the polster, to quantify protrusion orientation. Position of the ablation is indicated by a white dashed line on experimental images, and a red line on schematics; arrowheads mark actin rich protrusions; white arrow indicates direction of the Animal Pole (AP). (B) Axial speed of polster cells, tracked by H2B-mCherry labelling of their nucleus, $n=8$ to 10 embryos, $149 \pm 11$ quantified cells per embryos. Gray bars indicate paired statistical tests on embryos before and after ablation. (C) Orientation of actin rich protrusions in Lifeact-mCherry labelled cells. Numbers of quantified cells were respectively 27 in 4 embryos; 24 in 4 embryos; 17 in 3 embryos; 34 in 5 embryos; and 24 in 3 embryos. (B-C) Schematics indicate the position of ablation; yellow and orange brackets indicate the region of the polster quantified after ablation.

Figure 3: Polster oriented migration requires contact with posterior axial mesoderm. (A) Removal of the polster revealed by in situ hybridization for gsc (red) and tbxta (blue) or fluorescence in the $T g$ (gsc:GFP) line. The blue arrow and thin white line mark the former polster position; red lines mark posterior axial mesoderm. (B) Transplantation of $59 \pm 51$ polster cells, $106 \pm 43 \mu \mathrm{m}$ ahead of the axis. The thin white line delineates transplanted cells; horizontal line marks the initial position of the rear of the transplanted group. (C) Trajectories, (D) axial speed (average of all transplanted cells in each embryo, $n=6$ embryos) and ( $E$ ) actin rich protrusion orientations ( $n=15$ Lifeact-mCherry labelled cells in 5 embryos) of transplanted cells. Gray bars indicate paired statistical tests.

Figure 4: Orientation of polster cells requires active migration of the following axial mesoderm. (A) Replacement of the polster by a wild-type (WT) polster, in control embryos or embryos in which axis extension is genetically slowed down. Axial speed of replaced polster cells (red boxes) and of the front of the following axial mesoderm (blue boxes) were measured. White line marks transplanted polster cells and red line marks following axial mesoderm. (B) Replacement of the polster by a WT polster or a DN-Rac1 expressing polster, of varying size. Lines mark the initial position of the polster front. Axial speed of polster cells is plotted as a function of polster size. Unmanipulated WT embryos have been quantified for comparison (blue circle). (C) Protrusion orientation of WT cells in WT (33 
cells in 7 embryos) and DN-Rac1 (29 cells in 4 embryos) polsters. Inlay indicates the average number of protrusions per frame for each condition. (D) Protrusion orientation of WT cells transplanted among a small group of WT ( 25 cells in 6 embryos), DN-Rac1 (19 cells in 7 embryos) or Mo E-cadherin (11 cells in 6 embryos) cells labelled with H2B-mCerulean, in front of a WT polster. Inlay indicates the average number of protrusions per frame for each condition. White lines mark the endogenous polster of host embryos.

Figure 5: Lateral mesoderm can drive polster cell migration. (A) H2B-mCherry expressing polster cells (strong green, red nuclei) were transplanted ahead of the lateral mesoderm ( $T g(t b \times 16: G F P)$; faint green). A thin white line delineates polster cells; green line marks the lateral mesoderm anterior edge; horizontal line marks the position of the rear of the polster cell group upon contact with the lateral mesoderm. (B) Polster cell trajectories before and after contact with the lateral mesoderm of a typical experiment. (C) Axial speed ( $n=8$ embryos) of transplanted polster cells before and after contact with the lateral mesoderm. (D) Protrusion orientation of Lifeact-mCherry expressing polster cells (red labelled actin) transplanted along with other polster cells expressing H2B-mCerulean (green with blue nuclei) in front of the lateral mesoderm, quantified before and after contact ( 22 red cells in 3 embryos).

Figure 6: $\alpha$-Catenin mechanosensation and Vinculin are required for polster cell orientation. Actin rich protrusions of Lifeact-mCherry expressing cells transplanted in a WT polster. Arrowheads mark actin rich protrusions. Mo Ctrl: $n=28$ cells in 6 embryos for panel (B) and $n=37$ cells in 10 embryos for panel (C), Mo $\alpha$-Catenin: $n=25$ cells in 6 embryos, rescue $\alpha$-Catenin: $n=22$ cells in 4 embryos, rescue $\alpha$-Catenin $\triangle$ VBS: $n=34$ cells in 8 embryos, rescue $\alpha$-CateninL344P: $n=30$ cells in 8 embryos, Mo Vinculin $a \& b n=29$ cells in 9 embryos, rescue Vinculin $a \& b n=24$ cells in 6 embryos.

Figure 7: Guidance by followers. (A, B) Cellular Potts models testing different scenarios. (A) Polster cells are given a Run and Tumble behaviour, fitted to observations of isolated polster cells (see Figure S4). On their own, polster cells tend to disperse. When followed by axial mesoderm, they progress towards the animal pole, but mix with axial mesoderm. Adding mechanical sensitivity to neighbours migrating towards them (guidance by followers) is sufficient to account for the collective oriented migration of polster cells (and all experimental observations, see Movie S7). (B) If both polster cells and axial mesoderm are given oriented migrations towards the animal pole, differences in their speed will induce axis disruption. On the contrary, when polster cells are oriented by followers, polster speed spontaneously adjusts to axis speed. This stems from polster cell orientation being dependent on posterior cell speed (see Figure S5B). (C) Model of guidance of polster cells. Cells 
perceive mechanical stimuli generated by the active migration of their neighbours, and orient their protrusive activity accordingly. This leads to propagation of the directional information through the entire group.

\section{STAR Methods}

\section{Resource availability}

Lead contact

Further information and requests for resources and reagents should be directed to and will be fulfilled by the lead contact, Nicolas David (Nicolas.david@polytechnique.edu)

\section{Materials availability}

This study did not generate new unique reagents.

\section{Data and code availability}

The Morpheus model generated during this study was deposited in the public model repository under MorpheusModelID:M0006 (https://identifiers.org/morpheus/M0006). Custom Matlab routines used to process cell tracks are available upon request.

\section{Experimental model}

Zebrafish embryos were obtained by natural spawning of $A B, T g(t b \times 16: E G F P)$ and $T g(-$ 1.8gsc:GFP)m/1 adult fishes (Doitsidou et al., 2002; Wells et al., 2011). All animal studies were approved by the Ethical Committee $\mathrm{N}^{\circ} 59$ and the Ministère de l'Education Nationale, de I'Enseignement Supérieur et de la Recherche under the file number APAFIS\#158592018051710341011v3.

\section{Method details}

\section{In Situ Hybridization}

Whole-mount colour and fluorescent In Situ Hybridization were performed following standard protocols (Hauptmann and Gerster, 1994) using goosecoid, tbxta and cts/b probes (SchulteMerker et al., 1994; Stachel et al., 1993; Thisse et al., 1994).

\section{Embryo injection}

Translation blocking morpholinos (Gene Tool LLC Philomath) and concentration used were:

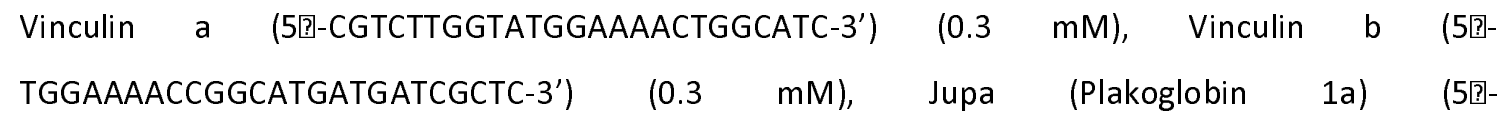


GAGCCTCTCCCATGTGCATTTCCAT-3') (0.4 mM) (Martin et al., 2009), Jupb (Plakoglobin 1b) (50?CCTCACTCATTTGCAGTGACATCAC-3') (0.1 mM), E-cadherin (5? ? (0.3 mM) (Babb and Marrs, 2004), $\alpha$-Caten in (50?-TAATGCTCGTCATGTTCCAAATTGC-3') (0.1 mM) (Han et al., 2016), Sox32 (5?-CAGGGAGCATCCGGTCGAGATACAT-3') (0.3 mM) (Dickmeis et al., 2001), and standard control (5? ??-CCTCTTACCTCAGTTACAATTTATA-3') (0.1 to $0.3 \mathrm{mM}$ ).

Capped sense mRNA were synthesized from pCS2+ constructs with mMessage mMachine SP6 kit (Thermo Fischer). Constructs and concentrations used were: Histone $2 \mathrm{~B}$ mCherry ( 30 to $50 \mathrm{ng} / \mu \mathrm{l}$ ), Histone2B-mCerulean (30 to $50 \mathrm{ng} / \mu \mathrm{l}$ ), Lifeact-mCherry (30 to $50 \mathrm{ng} / \mu \mathrm{l}$ ), Taram- $\mathrm{A}^{*}(0.6 \mathrm{ng} / \mu \mathrm{l}$ ),

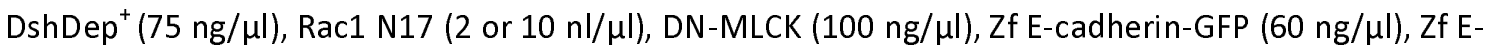

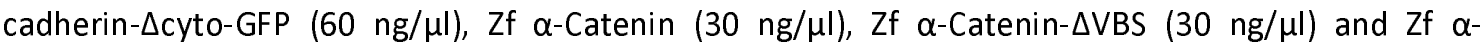
Catenin-L344P (30 ng/ $\mu \mathrm{l})$, Zf Vinculin a-GFP $(25 \mathrm{ng} / \mu \mathrm{l})$, Zf Vinculin b-GFP $(25 \mathrm{ng} / \mu \mathrm{l})$.

To label and/or affect the whole embryo, $5 \mathrm{nl}$ were injected at the one-cell stage. For donor embryos for cell transplantation, $1.5 \mathrm{nl}$ were injected in one cell at the four-cell stage.

\section{Cell transplantation and microsurgery}

Cell transplantations were performed as described in (Boutillon et al., 2018). Cells transplanted within the polster were taken from the shield of a $\operatorname{Tg}(g s c: G F P)$ donor and transplanted to the shield of a $T g$ (gsc:GFP) host at 6 hpf. Identity of transplanted cells was then assessed by their GFP expression. Cells transplanted out of the polster (animal pole, lateral side, ahead of the polster) were taken from donor embryos injected, in one cell out of four, with Tar* mRNA and Sox32 morpholino, so as to impose a polster identity (Dumortier et al., 2012). For single cell transplant, donor embryos were dissociated at shield stage in Ringer's without calcium solution prior to transplantation. Removal of the polster was performed in $T g$ (gsc:GFP) embryos, by aspiration with a large homemade glass pipette. The polster was identified on morphological criteria, confirmed by in situ hybridization against $c t s / b$, a marker for polster identity, and gsc, a marker for prechordal plate (Figures $3 \mathrm{~A}$ and S1).

\section{Embryo imaging}

Imaging of embryos for protrusion quantification was done on an inverted TCS SP8 confocal microscope (Leica) equipped with environmental chamber (Life Imaging Services) at $28^{\circ} \mathrm{C}$ using a $\mathrm{HC}$ PL APO 40x/1.10 W CS2 objective (Leica). Imaging of embryos for cell migration quantification was done under an upright TriM Scope II (La Vision Biotech) two-photon microscope equipped with an environmental chamber (okolab) at $28^{\circ} \mathrm{C}$ and a XLPLN25XWMP2 (Olympus) 25x water immersion objective or on the inverted TCS SP8 microscope (Leica) using a HCX PL Fluotar 10x/0.3 objective 
(Leica). Injected embryos were mounted in $0.2 \%$ agarose in embryo medium between $60 \%$ and $70 \%$ epiboly (6.5-7.5 hpf). Embryos were imaged between 30 and 60 minutes, every one to three minutes.

\section{Laser ablation}

Laser ablation experiments were performed under the TriM Scope II microscope (La Vision Biotech) equipped with a femtosecond Mai Tai HP DeepSee laser (Spectra Physics) and an Insight DeepSee (Spectra Physics) laser. Embryos were imaged every minute for 10 to 15 minutes prior to ablation. GFP was excited by the Mai Tai laser set to $920 \mathrm{~nm}$ wavelength and mCherry by the Insight laser set to $1160 \mathrm{~nm}$. Ablations were performed with the Mai Tai laser at $820 \mathrm{~nm}$ and exit power at 0.3 $m W$. Such an exit power allowed efficient ablation with very good axial confinement. The region to be ablated was defined as an $X Y$ ROI, and selectively illuminated using an EOM. To perform 3D ablations, laser treatment was performed on different focal planes, separated by 10 to 15 microns, starting with deeper planes. To compensate for the loss of energy in deeper planes, the number of treatment repeats was modulated with depth. Efficiency of the ablation was assessed by the absence of GFP fluorescence and the presence of cellular debris, and later confirmed by observation of locally modified cell behaviour. Embryos were imaged for 30 to 40 minutes following ablation. The polster was identified on morphological criteria and distance to the front, confirmed by in situ hybridization against $c t s / b$, a marker for polster identity (Figure S1D).

\section{Illustrations}

Images were processed with FIJI. Figures were assembled with Adobe InDesign, movies with Adobe Premiere Pro.

\section{Model simulations}

To model cell motility and cell-cell interactions, we chose a Cellular Potts Model (CPM) since the CPM allows for arbitrary cell shapes, spatially resolved cell-cell contact interfaces and stochasticity in cell movement (Graner and Glazier, 1992). Multiple modelling and simulation frameworks for CPM exist including Chaste (Mirams et al., 2013; Pitt-Francis et al., 2009), CompuCell3D (Swat et al., 2012) and Morpheus (Starruß et al., 2014) which are free, open-source software. We have chosen Morpheus because of its user-friendly interface and its transparent separation of the solver code from the computational model description in the domain-specific language MorpheusML. The model description file was deposited in the public model repository 
under MorpheusModelID:M0006 (https://identifiers.org/morpheus/M0006) which renders our multicellular simulations readily reproducible and extensible following the FAIR principles.

The simulations were performed on an elongated spatial domain with $500 \times 1500$ grid nodes of a two-dimensional hexagonal lattice with periodic boundary conditions. Left- and right-flanking static obstacles left a central channel of 200 nodes width for the cells to migrate into. These obstacles were used to mimic lateral confinement by paraxial mesoderm. The spatial unit is chosen as $1 \mu \mathrm{m}$ per grid interval and the temporal unit as 1 min per time step. Monte Carlo step duration was chosen as $0.1 \mathrm{~min}$ to allow thousands of potential updates per lattice node during the simulated time span. Cell shape is controlled by a target area of experimentally measured $326 \mu \mathrm{m}^{2}$ (average of 360 experimental measures) and a target circumference taken from the isoareal circle. Both constraints enter the Hamiltonian with equal Lagrange multipliers of 1 (Graner and Glazier, 1992). Axial mesoderm cells (yellow in simulations) are given a directed motion targeted at the animal pole, the speed of which is modulated by varying the strength parameter of the Directed Motion plugin in Morpheus (Starruß et al., 2014). Unless specified, polster cells (green in simulations) are given a Runand-Tumble motility with uniform reorientation probability of the target direction, a nondimensional scaling factor "Run_duration_adjustment" of the Gamma-distributed probabilistic waiting times for reorientation events, i.e. Run_duration_adjustment * $\Gamma(0.5,5 \mathrm{~min})$ with a mean run time of Run_duration_adjustment*0.5*5 min, and a tunable Lagrange multiplier "motion_strength" that scales motion speed. The two parameter values for Run_duration_adjustment and motion_strength were estimated from experimental data of single cell trajectories (see below). In addition to the Run-and-Tumble motility, mechanical orientation of polster cells was simulated using the PyMapper plugin. At fixed time steps of $1 \mathrm{~min}$, for each cell, neighbours are detected on 50 membrane points. For each neighbour, the angle between its velocity vector and the direction towards the considered cell is computed. If below a threshold "max_angle" (i.e. neighbour migrating towards the considered cell), the velocity vector of the neighbour is used as the new direction of the considered cell in the Directed Motion plugin. In case of several migrating neighbours, the direction vector is an average of their velocity vectors, weighed by the size of cell-cell contacts. In most simulations, 400 cells are initialized. An initial phase of $20 \mathrm{~min}$ without motility is used to equilibrate cell shapes and cell packing (not shown on the movies). Based on their antero-posterior position, cells are then given an identity, and the corresponding motility properties. Main parameters are summarized in Table S1.

For simulations with Contact Inhibition of Locomotion, identical parameters were used (Monte Carlo step duration, target area and circumference) and polster cells were given the same Run and Tumble behaviour. Instead of adding mechanical orientation, a CIL behaviour was added. Briefly, a membrane property is used to detect contact with neighbouring cells. The vector between 
the cell center and the contact point is measured and the opposite vector is added to the current cell direction, with a tunable Lagrange multiplier "cil_strength". Simulations were performed on a square domain with $1000 \times 1000$ grid nodes of a two-dimensional hexagonal lattice with periodic boundary conditions. Two groups of 40 cells were initialized, their centres $120 \mu \mathrm{m}$ apart.

\section{Parameter estimation}

In order to fit the baseline cell motility parameters to experimentally observed single cell trajectory data, we define a distance function between the observed and simulated summary statistics.

$$
d=\sum_{i} w_{i}^{M S D} *\left|M S D_{i}-m s d_{i}\right|+\sum_{i<21 \min } w_{i}^{D A C} *\left|D A C_{i}-d a c_{i}\right|
$$

Here, MSD is the mean square displacement and DAC the direction autocorrelation function (Gorelik and Gautreau, 2014). Capital variables represent the experimental measurements, small letters represent the model observables. We calculate the sum of weighted (see below) differences between the ensemble ( $n \sim 200$ ) means of MSD and DAC at the time points $i \in\{0 \mathrm{~min}, 3 \mathrm{~min}, 6 \mathrm{~min}$, $9 \mathrm{~min}, 12 \mathrm{~min}, 15 \mathrm{~min}, 18 \mathrm{~min}, 21 \mathrm{~min}\}$. We optimize this distance function $d$ employing the FitMultiCell software (https://fitmulticell.gitlab.io), which is a free, open-source Python tool embedding stochastic, multi-cellular Morpheus simulations in the highly parallel and unbiased Approximate Bayesian Computation - Sequential Monte Carlo (ABC-SMC) algorithm implemented by the computational framework pyABC (Klinger et al., 2018; Schälte and Hasenauer, 2020). FitMultiCell concurrently evaluates the model and distance measure $d$ for trial parameter sets drawn from the evolving probability distribution across the search space, started from a uniform prior distribution. The distance measure $d$ is then minimized over successive epochs by only accepting parameter sets with $d$ below a gradually decreasing acceptance threshold $\varepsilon$. The pyABC meta-parameters for parameter sample number was set to 200 accepted trial parameter sets per epoch. The computations were run on the high performance computing cluster of ZIH at TU Dresden with 4 CPUs used per task, $2.5 \mathrm{~GB} /$ core. The approximate run time for 10 epochs was $20 \mathrm{hrs}$.

The weights $w_{i}$ were chosen adaptively in pyABC to account for the different scales of MSD and DAC and two sets of optimization epochs were concatenated. First, a uniform prior distribution in the broad interval $[0.01,10]$ was chosen for each fit parameter and the adaptive-weight scheme of pyABC readjusted the $w_{i}$ after each of 10 epochs. To avoid convergence problems for later epochs due to fluctuating weights, we used the posterior distributions of this first set of epochs as the prior for the second set of epochs, i.e. Run_duration_adjustment: $[0.05,4]$, motion_strength: [0.1, 2], advection_velocity: $[0.05,3]$. The weights $w_{i}$ of the second set of epochs were again adaptively 
adjusted by pyABC but just initially and then kept fixed for the remaining 12 epochs. Convergence was judged by arriving at the plateau of the acceptance threshold $\varepsilon$ in the ABC-SMC algorithm, see Figure S4. The following point estimate for the fitted model parameters and their confidence intervals were obtained (Figure S4A):

Run_duration_adjustment: $0.76, \mathrm{Cl}:[0.18,1.95]$

motion_strength: $0.50, \mathrm{Cl}:[0.28,1.00]$

advection_velocity: $1.42, \mathrm{Cl}:[0.24,2.20]$

The parameter advection_velocity was used to overlay a uniform translation onto all cells, capturing potential drag forces by the overlying ectoderm. Such common translation reproduces the experimentally observed baseline of $20 \%$ in DAC (Figure S4D).

\section{Quantification and statistical analysis}

Image analysis

Cell movements were quantified by tracking cell nuclei, labelled with H2B-mCherry, using IMARIS (Bitplane). Tracks were then processed using custom-made Matlab (Math Works) routines as described in (Dumortier et al., 2012). Axial mesoderm elongation was quantified by tracking migration of cells at its front. Actin-rich protrusions were quantified on Lifeact-mCherry expressing cells. Protrusion orientation was manually measured as the angle between protrusion axis and the animal-vegetal axis, using ImageJ (FIJI), as described in (Boutillon et al., 2018).

\section{Statistical analysis}

All statistical analyses were performed in $\mathrm{R}$ ( $\mathrm{R}$ project). Cell migration absolute and axial speed were averaged over cells and embryos, and compared using Wilcoxon tests. When relevant, paired Wilcoxon tests were performed, as indicated on the corresponding boxplots. Protrusion angle distributions and frequency were compared using linear mixed models taking into account the fact that measurements are not independent (several measurements for each cell, several cells for each embryo).

To serve as controls for collision data (see Figure 1B), 100 bootstrapped datasets were generated by randomly picking, for 80 cells, one time-step where the cell moves freely (no collision) and measuring the angle between incident and efferent vectors ( $26 \pm 13$ available times per cell). Each of these bootstrapped datasets, along with the combination of all, were compared to the angle of 
deflection upon collision using a Kolmogorov-Smirnov test. Only one bootstrapped dataset is statistically different, which is less than expected by chance with an $\alpha$ risk of $5 \%$.

\section{Key Resources Table}

\begin{tabular}{|c|c|c|}
\hline REAGENT or RESOURCE & SOURCE & IDENTIFIER \\
\hline \multicolumn{3}{|l|}{ Deposited data } \\
\hline Raw images & $\begin{array}{l}\text { This paper; Mendeley } \\
\text { data }\end{array}$ & $\begin{array}{l}\text { http://dx.doi.org/10.1 } \\
\text { 7632/7ckg3p8d7d.1 }\end{array}$ \\
\hline \multicolumn{3}{|l|}{ Experimental models: Organisms/strains } \\
\hline $\begin{array}{l}\text { Zebrafish } \mathrm{AB} \\
\text { Zebrafish } T g(-1.8 g s c: G F P) m l 1\end{array}$ & $\begin{array}{l}\text { Doitsidou et al., } \\
2002\end{array}$ & $\begin{array}{l}\text { ZDB-ALT-051004- } \\
2\end{array}$ \\
\hline Zebrafish $T g(t b x 16: E G F P)$ & Wells et al., 2011 & $\begin{array}{l}\text { ZDB- } \\
\text { TGCONSTRCT- } \\
110722-1\end{array}$ \\
\hline \multicolumn{3}{|l|}{ Oligonucleotides } \\
\hline $\begin{array}{l}\text { Morpholino Vinculin a: } \\
\text { CGTCTTGGTATGGAAAACTGGCATC }\end{array}$ & Gene Tools & This paper \\
\hline $\begin{array}{l}\text { Morpholino Vinculin b: } \\
\text { TGGAAAACCGGCATGATGATCGCTC }\end{array}$ & Gene Tools & This paper \\
\hline $\begin{array}{l}\text { Morpholino Jupa (Plakoglobin 1a): } \\
\text { GAGCCTCTCCCATGTGCATTTCCAT }\end{array}$ & Gene Tools & $\begin{array}{l}\text { ZDB-MRPHLNO- } \\
091103-3\end{array}$ \\
\hline $\begin{array}{l}\text { Morpholino Jupb (Plakoglobin 1b): } \\
\text { CCTCACTCATTTGCAGTGACATCAC }\end{array}$ & Gene Tools & This paper \\
\hline $\begin{array}{l}\text { Morpholino E-Cadherin: } \\
\text { TAAATCGCAGCTCTTCCTTCCAACG }\end{array}$ & Gene Tools & $\begin{array}{l}\text { ZDB-MRPHLNO- } \\
050421-2\end{array}$ \\
\hline $\begin{array}{l}\text { Morpholino } \alpha \text {-Catenin: } \\
\text { TAATGCTCGTCATGTTCCAAATTGC }\end{array}$ & Gene Tools & $\begin{array}{l}\text { ZDB-MRPHLNO- } \\
120206-2\end{array}$ \\
\hline $\begin{array}{l}\text { Morpholino Sox32: } \\
\text { CAGGGAGCATCCGGTCGAGATACAT }\end{array}$ & Gene Tools & $\begin{array}{l}\text { ZDB-MRPHLNO- } \\
\text { 051216-6 }\end{array}$ \\
\hline $\begin{array}{l}\text { Morpholino standard control: } \\
\text { CCTCTTACCTCAGTTACAATTTATA }\end{array}$ & Gene Tools & N/A \\
\hline \multicolumn{3}{|l|}{ Software and algorithms } \\
\hline ImageJ & Schneider et al., 2012 & $\begin{array}{l}\text { https://imagej.nih.go } \\
\text { v/ij/ }\end{array}$ \\
\hline $\begin{array}{l}\text { Matlab } \\
\text { IMARIS }\end{array}$ & $\begin{array}{l}\text { Math Works } \\
\text { Bitplane }\end{array}$ & \\
\hline $\mathrm{R}$ & & $\begin{array}{l}\text { https://www.r- } \\
\text { project.org// }\end{array}$ \\
\hline InDesign & Adobe & \\
\hline Premiere Pro & Adobe & \\
\hline Morpheus & Starruß et al., 2014 & $\begin{array}{l}\text { https://morpheus.gitl } \\
\text { ab.io/ }\end{array}$ \\
\hline Morpheus model & This paper & $\begin{array}{l}\text { https://identifiers.org/ } \\
\text { morpheus/M0006 }\end{array}$ \\
\hline
\end{tabular}




\section{Supplemental Information}

Table S1. Summary of model parameter values.

\begin{tabular}{|l|c|c|c|l|}
\hline Model parameter & Symbol & Value & Unit & Reference \\
\hline target cell area & $A_{0}$ & 326 & $\mu \mathrm{m}^{2}$ & experimental measurement \\
\hline target cell circumference & $C_{0}$ & $\sqrt{4 \pi A_{0}}$ & $\mu \mathrm{m}$ & free choice \\
\hline polster cell, motion strength & $\lambda_{1}$ & 0,5 & - & fitted to single cell data \\
\hline polster cell, mean run time & $T_{1}$ & $0,76^{*} 2,5$ & $\mathrm{~min}$ & fitted to single cell data \\
\hline $\begin{array}{l}\text { polster cell, advection } \\
\text { velocity }\end{array}$ & $v_{1}$ & 1,42 & $\frac{\mu \mathrm{m}}{\mathrm{min}}$ & fitted to single cell data \\
\hline maximum angle & $\alpha_{\max }$ & $\pi / 6$ & - & fitted to collective behaviour \\
\hline
\end{tabular}

Figure S1: Situation of polster cells and laser ablations, related to Figure 2. (A) Scheme of a gastrulating embryo at $70 \%$ epiboly in dorsal view; Im: lateral mesoderm; black arrow marks the direction of polster migration. Close-ups on the forming embryonic axis in Tg(gsc:GFP) embryo where axis is labelled in green, along with some endodermal cells (white line delineates the polster; red lines mark the posterior axial mesoderm; arrowheads point to some endodermal cells), and in a fluorescent in situ hybridization (FISH) for different antero-posterior markers of the axis (sagittal and dorsal views). Polster precursors expressing $c t s / b$ and notochord precursors expressing tbxta appear in green, prechordal plate progenitors expressing gsc appear in red. (B) Representative $\operatorname{Tg}(g s c: G F P$ ) embryo before and after laser ablation, here between the polster and the following mesoderm. Sagittal and dorsal views. Membranes are labelled in red by expression of mCherry-CAAX. Ablation is located between white arrowheads. YSL: yolk syncytial layer; EVL: enveloping layer. (C) Morphology and survival of control and ablated $T g$ (gsc:GFP) embryos at $24 \mathrm{hpf}$. The polster derivative, the hatching gland, is indicated by red arrowheads. (D) Fluorescent in situ hybridization for $c t s / b$ and tbxta on a representative embryo ablated at the interface between the polster and the posterior axial mesoderm. Position of the ablation is visible through red autofluorescent debris. Distance between the front of the polster and either the posterior edge of the ctslb domain, the position of ablations between the polster and following mesoderm, and of ablations within the following mesoderm.

Figure S2: Migration speed of polster cells after laser ablations, related to Figure 2. (A) 3D views of polster and axial mesoderm (green) migration, in a Tg(gsc:GFP) embryo expressing H2B-mCherry 
(red). Nuclei belonging to the polster are highlighted in magenta and tracked over time (see Movie S2). AP: animal pole. (B) Absolute speed of polster cells in control and ablated embryos, corresponding to the axial speeds presented in Figure 2. (C) Axial speed of the anterior and posterior parts of the polster after ablation in its middle. (B-C) Schematics indicate the position of ablation; yellow and orange brackets indicate the region of the polster quantified after ablation. Gray bars indicate paired statistical tests on embryos before and after ablation. (D) Axial speed of polster cells in embryos ablated between the polster and the posterior mesoderm, as a function of time $(n=6$ embryos). The moment of the ablation and the average moment of wound healing are indicated.

Figure S3: E-cadherin is required for polster cell orientation while Plakoglobin is not, related to Figure 6. (A) Polster cells injected with Lifeact-mCherry RNAs and control morpholino ( $n=23$ cells in 6 embryos), E-cadherin morpholino ( $\mathrm{n}=28$ cells in 6 embryos), E-cadherin morpholino and E-cadherin mRNA (resc. E-cadherin: $n=22$ cells in 4 embryos), or E-cadherin morpholino and E-cadherin $\Delta$ cyto (resc. E-cadherin $\Delta$ cyto: $n=34$ cells in 8 embryos) mRNA were transplanted in a wild-type polster. Orientation of actin rich protrusions (arrowheads) were quantified. (B) Polster cells injected with Lifeact-mCherry RNAs and a control Morpholino ( $n=26$ in 9 embryos) or Morpholinos targeting jupa and jupb ( $\mathrm{n}=29$ cells in 9 embryos) were transplanted in a wild-type polster. Orientation of protrusions were quantified. (C) Phenotypes at 24 and $72 \mathrm{hpf}$ of control uninjected embryos ( $n=37$ ) and embryos injected with jupa and jupb morpholinos ( $\mathrm{n}=47$ ). (D) Phenotypes at $24 \mathrm{hpf}$ of embryos injected with a control morpholino or Mo $\alpha$-Catenin, with or without mRNAs. (C-D) Number of analysed embryos are indicated above each bar.

Figure S4: Parameter estimation for Cellular Potts Model simulations, related to Figure 7. (A) Inference of model parameters from experimental data with ABC-SMC optimization using the FitMultiCell toolbox. Estimation results are shown as normalized two-parameter (top row) and oneparameter (bottom-row) kernel density estimates of the posterior parameter distributions. The red dot (top row) and red-dashed line (bottom row) represent the weighted median (for details see Methods, Parameter estimation). (B) Evolution of the acceptance threshold through optimization epochs. (C) Mean Square Displacement and (D) Differential Autocorrelation of experimental and simulated data with estimated parameters.

Figure S5: Comparison of simulated and experimental data, related to Figure 7. (A) Close-ups from simulated and experimental laser ablations, revealing two features we first noticed in simulations and then identified in experimental data: a backward movement of the cells at the posterior edge of the isolated group and a tendency of the most anterior cells to migrate out of the group. Both 
features were quantified in experimental data. (B) Orientation of cell movements when speed of following cells (axis) varies. Experimental data are those presented in Figure 4A, and correspond to an unmanipulated polster (ctrl), or a wild-type poster transplanted in either a wild-type embryo, a Dsh-DEP+ injected embryo or a DN-Rac1 injected embryo. Simulations were performed with varying speed of axial cells, by modulating the Lagrange multiplier modulating their movement (Imposed Motion Strength). Distribution of orientations are plotted in the different situations as cumulative plots. The percentage of movements oriented towards the animal pole $(<45 \%)$ is plotted as a function of the measured speed of the axis.

Movie S1: Polster cells do not exhibit CIL or CoA behaviour, related to Figure 1. Mixing assay: two differently labelled groups of polster cells were transplanted side by side at the animal pole of a host embryo. Their overlap (yellow) was measured after $90 \mathrm{~min}$. Green is GFP, red is Lifeact-mCherry. Collision assay: example of a collision between polster cells transplanted the animal pole of a host embryo. Nuclei, labelled with Histone2B-mCherry were tracked (white ellipsoid). Trajectories before and after contact are highlighted, respectively in cyan and yellow. One cell ahead of the polster: one polster cell, labelled with Lifeact-mCherry (red), was transplanted ahead of the polster (green). Two nearby groups: two differently labelled groups of polster cells were transplanted $160 \mu \mathrm{m}$ apart, to see if they attract each other. Areas covered by each group are highlighted in green and red at times 0 and $90 \mathrm{~min}$. Green is GFP, red is Lifeact-mCherry. Scale bar is $50 \mu \mathrm{m}$ for all four movies.

Movie S2: Four-dimensional tracking of polster nuclei, related to Figure 2. Nuclei of a $T g$ (gsc:GFP) embryo were labelled with Histone2B-mCherry. Z-stacks were acquired every minute. Nuclei of polster cells, identified by GFP expression and morphological criteria, are highlighted in magenta and 3D-tracked in time. Animal pole is to the top. Scale bar is $50 \mu \mathrm{m}$.

Movie S3: Laser ablations, related to Figure 2. Maximum projections of z-stacks acquired every minute in gastrulating $T g$ (gsc:GFP) embryos. Embryos were first imaged for 10 minutes. A laser ablation was then performed at the location indicated in each panel by the red bar on the movie and on the small schematic. Embryos were then imaged for 40 minutes. Animal pole is to the top. Scale bar is $50 \mu \mathrm{m}$.

Movie S4: Contact with posterior axial mesoderm drives polster cell migration, related to Figure 3. The polster of an unlabelled $T g$ (gsc:GFP) host was removed, while the following axial mesoderm was left intact (dim green). Polster cells from a Tg(gsc:GFP) donor (bright green), labelled with Histone2BmCherry (red nuclei), were transplanted ahead of the axial mesoderm. Z-stacks were acquired every 2-minute, maximum projections are shown here. Before contact with the extending axial mesoderm, 
transplanted polster cells do not display directional migration. After contact, the group of polster cells migrates in the same direction as the extending axial mesoderm. Animal pole is to the top. Scale bar is $50 \mu \mathrm{m}$.

Movie S5: Contact with the lateral mesoderm can orient polster cell migration, related to Figure 5. Polster cells from a $\mathrm{Tg}(g s c: G F P)$ donor (bright green), labelled with Histone2B-mCherry (red nuclei), were transplanted ahead of the lateral mesoderm of a $T g(t b x 16: G F P)$ embryo (dim green). Z-stacks were acquired every 3-minute, maximum projections are shown here. Trajectories of representative cells are highlighted. Before contact with the lateral mesoderm, polster cells tend to spread (cyan tracks), while after contact they align with the lateral mesoderm (yellow tracks). Animal pole is to the top. Scale bar is $50 \mu \mathrm{m}$.

Movie S6: $\alpha$-Catenin and Vinculin mediated mechanosensation is required for polster cell orientation, related to Figure 6. Polster cells injected with morpholino (Mo) or morpholino and mRNA (Resc.) and labelled with Lifeact-mCherry were transplanted in the polster of wild-type embryos and acquired in 3D over time. Maximum projections are shown. Time-interval between frames is, from left to right, top to bottom: $90 \mathrm{~s}, 60 \mathrm{~s}, 120 \mathrm{~s}, 60 \mathrm{~s}, 60 \mathrm{~s}, 120 \mathrm{~s}$ and $90 \mathrm{~s}$. Animal pole (AP) is to the top. Scale bar is $20 \mu \mathrm{m}$.

Movie S7: Simulations of polster migration, related to Figure 7. Mechanical orientation (guidance by followers) can account for directed migration of the polster: movies of the three scenarios presented on Figure 7A. Simulations reproduce experimental observations: polster cells are given a Run and Tumble behaviour and mechanical sensitivity to neighbours migrating towards them (guidance by followers). When isolated cells, polster cells tend to disperse, an equivalent experimental condition (polster cells transplanted to the animal pole of a host embryo) is presented for comparison. Simulating laser ablations at the front does not affect polster migration, as observed (Figure 2 and Movie S3). Simulating laser ablation at the interface between polster and following mesoderm halts progression of the polster till wound healing, as observed (Figure 2 and Movie S3). Each cell responding to all its neighbours cannot account for experimental observations: a model in which each cell responds to any neighbour (migrating towards or away from it) can account for the directed migration of the polster (left movie) but cannot account for the behaviour of an isolated polster (center movie) or for the behaviour after laser ablation at the interface between the polster and the following mesoderm (right movie). Mechanical orientation (guidance by followers) provides robustness and coordination: movies of the three scenarios presented on Figure 7B. Coordination of the progression of the polster and of the following mesoderm can be achieved by giving both tissues a directed migration (left movie). However, such a system is very sensitive to differences in cell speed 
(center movie). On the opposite, when polster cells are mechanically oriented, polster speed adjusts to the speed of the following mesoderm, ensuring axis continuity. This stems from polster cells being less oriented when followed by slow axial cells (see Figure S5B).

\section{References}

Babb, S.G., and Marrs, J.A. (2004). E-cadherin regulates cell movements and tissue formation in early zebrafish embryos. Dev. Dyn. 230, 263-277, 10.1002/dvdy.20057.

Barone, V., Lang, M., Krens, S.F.G., Sikora, M., Guet, C., and Heisenberg, C. (2017). An Effective Feedback Loop between Cell-Cell Contact Duration and Morphogen Signaling Determines Cell Fate. Dev. Cell 198-211, 10.1016/j.devcel.2017.09.014.

Bazellières, E., Conte, V., Elosegui-Artola, A., Serra-Picamal, X., Bintanel-Morcillo, M., Roca-Cusachs, P., Muñoz, J.J., Sales-Pardo, M., Guimerà, R., and Trepat, X. (2015). Control of cell-cell forces and collective cell dynamics by the intercellular adhesome. Nat. Cell Biol. 17, 409-420, 10.1038/ncb3135.

Behrndt, M., and Heisenberg, C.-P. (2012). Spurred by Resistance: Mechanosensation in Collective Migration. Dev. Cell 22, 3-4, 10.1016/j.devcel.2011.12.018.

Blanco, M.J., Barrallo-Gimeno, A., Acloque, H., Reyes, A.E., Tada, M., Allende, M.L., Mayor, R., and Nieto, M.A. (2007). Snail1a and Snail1b cooperate in the anterior migration of the axial mesendoderm in the zebrafish embryo. Development 134, 4073-4081, 10.1242/dev.006858.

Borrell, V., and Marín, O. (2006). Meninges control tangential migration of hem-derived Cajal-Retzius cells via CXCL12/CXCR4 signaling. Nat. Neurosci. 9, 1284-1293, 10.1038/nn1764.

Bosze, B., Ono, Y., Mattes, B., Sinner, C., Gourain, V., Thumberger, T., Tlili, S., Wittbrodt, J., Saunders, T.E., Strähle, U., et al. (2020). Pcdh18a regulates endocytosis of E-cadherin during axial mesoderm development in zebrafish. Histochem. Cell Biol. 10.1007/s00418-020-01887-5.

Boutillon, A., Giger, F.A., and David, N.B. (2018). Analysis of In Vivo Cell Migration in Mosaic Zebrafish Embryos. Methods Mol. Biol. 1749, 213-226, 10.1007/978-1-4939-7701-7_16.

Čapek, D., Smutny, M., Tichy, A.-M., Morri, M., Janovjak, H., and Heisenberg, C.-P. (2019). Lightactivated Frizzled7 reveals a permissive role of non-canonical wnt signaling in mesendoderm cell migration. Elife 8, 1-56, 10.7554/elife.42093.

Carmona-Fontaine, C., Matthews, H.K., Kuriyama, S., Moreno, M., Dunn, G.A., Parsons, M., Stern, C.D., and Mayor, R. (2008). Contact inhibition of locomotion in vivo controls neural crest directional migration. Nature 456, 957-961, 10.1038/nature07441.

Carmona-Fontaine, C., Theveneau, E., Tzekou, A., Tada, M., Woods, M., Page, K.M., Parsons, M., Lambris, J.D., and Mayor, R. (2011). Complement fragment C3a controls mutual cell attraction during collective cell migration. Dev. Cell 21, 1026-1037, 10.1016/j.devcel.2011.10.012.

Das, D., Jülich, D., Schwendinger-Schreck, J., Guillon, E., Lawton, A.K., Dray, N., Emonet, T., O’Hern, C.S., Shattuck, M.D., and Holley, S.A. (2019). Organization of Embryonic Morphogenesis via Mechanical Information. Dev. Cell 49, 829-839.e5, 10.1016/j.devcel.2019.05.014.

Das, T., Safferling, K., Rausch, S., Grabe, N., Boehm, H., and Spatz, J.P. (2015). A molecular mechanotransduction pathway regulates collective migration of epithelial cells. Nat. Cell Biol. 17, 276-287, 10.1038/ncb3115. 
Dickmeis, T., Mourrain, P., Saint-Etienne, L., Fischer, N., Aanstad, P., Clark, M., Str??hle, U., Rosa, F.F.M.F., Strähle, U., and Rosa, F.F.M.F. (2001). A crucial component of the endoderm formation pathway, CASANOVA, is encoded by a novel sox-related gene. Genes Dev. 15, 1487-1492, 10.1101/gad.196901.

Doitsidou, M., Reichman-Fried, M., Stebler, J., Köprunner, M., Dörries, J., Meyer, D., Esguerra, C. V, Leung, T., and Raz, E. (2002). Guidance of primordial germ cell migration by the chemokine SDF-1. Cell 111, 647-659, doi.org/10.1016/S0092-8674(02)01135-2.

Dumortier, J.G., Martin, S., Meyer, D., Rosa, F.M., and David, N.B. (2012). Collective mesendoderm migration relies on an intrinsic directionality signal transmitted through cell contacts. Proc. Natl. Acad. Sci. U. S. A. 109, 16945-16950, 10.1073/pnas.1205870109.

Giger, F.A., and David, N.B. (2017). Endodermal germ-layer formation through active actin-driven migration triggered by N-cadherin. Proc. Natl. Acad. Sci. 114, 10143-10148, 10.1073/pnas.1708116114.

Glickman, N.S.S., Kimmel, C.B., Jones, M.A., and Adams, R.J. (2003). Shaping the zebrafish notochord. Development 130, 873-887, 10.1242/dev.00314.

Gorelik, R., and Gautreau, A. (2014). Quantitative and unbiased analysis of directional persistence in cell migration. Nat. Protoc. 9, 1931-1943, 10.1038/nprot.2014.131.

Graner, F., and Glazier, J.A. (1992). Simulation of biological cell sorting using a two-dimensional extended Potts model. Phys Rev Lett 69, 2013-2016.

Haeger, A., Wolf, K., Zegers, M.M., and Friedl, P. (2015). Collective cell migration: guidance principles and hierarchies. Trends Cell Biol. 25, 556-566, 10.1016/j.tcb.2015.06.003.

Han, M.K.L., Hoijman, E., Nöel, E., Garric, L., Bakkers, J., and de Rooij, J. (2016). $\alpha$ E-catenin-dependent mechanotransduction is essential for proper convergent extension in zebrafish. Biol. Open 5, 1461$1472,10.1242$ /bio.021378.

Hauptmann, G., and Gerster, T. (1994). Two-color whole-mount in situ hybridization to vertebrate and Drosophila embryos. Trends Genet. 10, 266.

Heisenberg, C.-P., Tada, M., Rauch, G.-J.J., Saúde, L., Concha, M.L., Geisler, R., Stemple, D.L., Smith, J.C., Wilson, S.W., Saude, L., et al. (2000). Silberblick/Wnt11 mediates convergent extension movements during zebrafish gastrulation. Nature 405, 76-81, 10.1038/35011068.

Hirata, H., Dobrokhotov, O., and Sokabe, M. (2020). Coordination between Cell Motility and Cell Cycle Progression in Keratinocyte Sheets via Cell-Cell Adhesion and Rac1. IScience 23, 101729, 10.1016/j.isci.2020.101729.

Hoffman, B.D., and Yap, A.S. (2015). Towards a Dynamic Understanding of Cadherin-Based Mechanobiology. Trends Cell Biol. 25, 803-814, 10.1016/j.tcb.2015.09.008.

Huveneers, S., Oldenburg, J., Spanjaard, E., van der Krogt, G., Grigoriev, I., Akhmanova, A., Rehmann, H., and de Rooij, J. (2012). Vinculin associates with endothelial VE-cadherin junctions to control force-dependent remodeling. J. Cell Biol. 196, 641-652, 10.1083/jcb.201108120.

Jurado, J., de Navascués, J., and Gorfinkiel, N. (2016). $\alpha$-Catenin stabilises Cadherin-Catenin complexes and modulates actomyosin dynamics to allow pulsatile apical contraction. J. Cell Sci. 129, 4496-4508, 10.1242/jcs.193268.

Kai, M., Heisenberg, C.-P., and Tada, M. (2008). Sphingosine-1-phosphate receptors regulate individual cell behaviours underlying the directed migration of prechordal plate progenitor cells during zebrafish gastrulation. Development 135, 3043-3051, 10.1242/dev.020396.

Kim, T.-J., Zheng, S., Sun, J., Muhamed, I., Wu, J., Lei, L., Kong, X., Leckband, D.E., and Wang, Y. 
(2015). Dynamic visualization of $\alpha$-catenin reveals rapid, reversible conformation switching between tension states. Curr. Biol. 25, 218-224, 10.1016/j.cub.2014.11.017.

Kimmel, C.B., Ballard, W.W., Kimmel, S.R., Ullmann, B., and Schilling, T.F. (1995). Stages of embryonic development of the zebrafish. Dev. Dyn. 203, 253-310, 10.1002/aja.1002030302.

Klinger, E., Rickert, D., and Hasenauer, J. (2018). pyABC: distributed, likelihood-free inference. Bioinformatics 34, 3591-3593, 10.1093/bioinformatics/bty361.

Kobielak, A., Pasolli, H.A., and Fuchs, E. (2004). Mammalian formin-1 participates in adherens junctions and polymerization of linear actin cables. Nat. Cell Biol. 6, 21-30, 10.1038/ncb1075.

Krens, S.F.G., Veldhuis, J.H., Barone, V., Čapek, D., Maître, J.-L., Brodland, G.W., and Heisenberg, C.-P. (2017). Interstitial fluid osmolarity modulates the action of differential tissue surface tension in progenitor cell segregation during gastrulation. Development 144, 1798-1806, 10.1242/dev.144964.

Ladoux, B., Nelson, W.J., Yan, J., and Mège, R.M. (2015). The mechanotransduction machinery at work at adherens junctions. Integr. Biol. (Camb). 7, 1109-1119, 10.1039/c5ib00070j.

Lauffenburger, D.A., and Horwitz, A.F. (1996). Cell migration: a physically integrated molecular process. Cell 84, 359-369, 10.1016/s0092-8674(00)81280-5.

Lecuit, T., and Yap, A.S. (2015). E-cadherin junctions as active mechanical integrators in tissue dynamics. Nat. Cell Biol. 17, 533-539, 10.1038/ncb3136.

Maître, J.-L., Berthoumieux, H., Krens, S.F.G., Salbreux, G., Jülicher, F., Paluch, E.K., and Heisenberg, C.-P. (2012). Adhesion Functions in Cell Sorting by Mechanically Coupling the Cortices of Adhering Cells. Science (80-. ). 10, 429-436, 10.1126/science.1225399.

Martin, E.D., Moriarty, M.A., Byrnes, L., and Grealy, M. (2009). Plakoglobin has both structural and signalling roles in zebrafish development. Dev. Biol. 327, 83-96, 10.1016/j.ydbio.2008.11.036.

Mirams, G.R., Arthurs, C.J., Bernabeu, M.O., Bordas, R., Cooper, J., Corrias, A., Davit, Y., Dunn, S.-J., Fletcher, A.G., Harvey, D.G., et al. (2013). Chaste: an open source C++ library for computational physiology and biology. PLoS Comput. Biol. 9, e1002970, 10.1371/journal.pcbi.1002970.

Mishra, A.K., Mondo, J.A., Campanale, J.P., and Montell, D.J. (2019). Coordination of protrusion dynamics within and between collectively migrating border cells by myosin II. Mol. Biol. Cell 30, 2490-2502, 10.1091/mbc.E19-02-0124.

Montero, J.-A., Kilian, B., Chan, J., Bayliss, P.E., and Heisenberg, C.-P. (2003). Phosphoinositide 3kinase is required for process outgrowth and cell polarization of gastrulating mesendodermal cells. Curr. Biol. 13, 1279-1289, 10.1016/S0960-9822(03)00505-0.

Montero, J.-A., Carvalho, L., Wilsch-Bräuninger, M., Kilian, B., Mustafa, C., Heisenberg, C.-P., and Wilsch-Brauninger, M. (2005). Shield formation at the onset of zebrafish gastrulation. Development 132, 1187-1198, 10.1242/dev.01667.

Myers, D.C., Sepich, D.S., and Solnica-Krezel, L. (2002). Convergence and extension in vertebrate gastrulae: cell movements according to or in search of identity? Trends Genet 18, 447-455.

Nguyen, T., and Mège, R.M. (2016). N-Cadherin and Fibroblast Growth Factor Receptors crosstalk in the control of developmental and cancer cell migrations. Eur. J. Cell Biol. 95, 415-426, 10.1016/j.ejcb.2016.05.002.

Nieset, J.E., Redfield, A.R., Jin, F., Knudsen, K.A., Johnson, K.R., and Wheelock, M.J. (1997). Characterization of the interactions of alpha-catenin with alpha-actinin and betacatenin/plakoglobin. J. Cell Sci. 110 ( Pt 8, 1013-1022.

Norden, C., and Lecaudey, V. (2019). Collective cell migration: general themes and new paradigms. Curr. Opin. Genet. Dev. 57, 54-60, 10.1016/j.gde.2019.06.013. 
Patel, N.G., Nguyen, A., Xu, N., Ananthasekar, S., Alvarez, D.F., Stevens, T., and Tambe, D.T. (2020). Unleashing shear: Role of intercellular traction and cellular moments in collective cell migration. Biochem. Biophys. Res. Commun. 522, 279-285, 10.1016/j.bbrc.2019.11.048.

Pézeron, G., Mourrain, P., Courty, S., Ghislain, J., Becker, T.S., Rosa, F.M., and David, N.B. (2008). Live analysis of endodermal layer formation identifies random walk as a novel gastrulation movement. Curr. Biol. 18, 276-281, 10.1016/j.cub.2008.01.028.

Pitt-Francis, J., Pathmanathan, P., Bernabeu, M.O., Bordas, R., Cooper, J., Fletcher, A.G., Mirams, G.R., Murray, P., Osborne, J.M., Walter, A., et al. (2009). Chaste: A test-driven approach to software development for biological modelling. Comput. Phys. Commun. 180, 2452-2471, 10.1016/j.cpc.2009.07.019.

Pokutta, S., Drees, F., Takai, Y., Nelson, W.J., and Weis, W.I. (2002). Biochemical and structural definition of the l-afadin- and actin-binding sites of alpha-catenin. J. Biol. Chem. 277, 18868-18874, 10.1074/jbc.M201463200.

Poujade, M., Grasland-Mongrain, E., Hertzog, A., Jouanneau, J., Chavrier, P., Ladoux, B., Buguin, A., and Silberzan, P. (2007). Collective migration of an epithelial monolayer in response to a model wound.

Roszko, I., Sawada, A., and Solnica-Krezel, L. (2009). Regulation of convergence and extension movements during vertebrate gastrulation by the Wnt/PCP pathway. Semin. Cell Dev. Biol. 20, 986997, 10.1016/j.semcdb.2009.09.004.

Scarpa, E., and Mayor, R. (2016). Collective cell migration in development. J. Cell Biol. 212, 143-155, $10.1083 /$ jcb.201508047.

Scarpa, E., Szabó, A., Bibonne, A., Theveneau, E., Parsons, M., and Mayor, R. (2015). Cadherin Switch during EMT in Neural Crest Cells Leads to Contact Inhibition of Locomotion via Repolarization of Forces. Dev. Cell 34, 421-434, 10.1016/j.devcel.2015.06.012.

Schälte, Y., and Hasenauer, J. (2020). Efficient exact inference for dynamical systems with noisy measurements using sequential approximate Bayesian computation. Bioinformatics 36, i551-i559, 10.1093/bioinformatics/btaa397.

Schulte-Merker, S., van Eeden, F.J., Halpern, M.E., Kimmel, C.B., and Nüsslein-Volhard, C. (1994). no tail ( $n \mathrm{tl})$ is the zebrafish homologue of the mouse T (Brachyury) gene. Development 120, 1009-1015.

Schumacher, L. (2019). Collective Cell Migration in Development. Adv. Exp. Med. Biol. 1146, 105-116, 10.1007/978-3-030-17593-1_7.

Seddiki, R., Narayana, G.H.N.S., Strale, P.-O., Balcioglu, H.E., Peyret, G., Yao, M., Le, A.P., Teck Lim, C., Yan, J., Ladoux, B., et al. (2018). Force-dependent binding of vinculin to $\alpha$-catenin regulates cell-cell contact stability and collective cell behavior. Mol. Biol. Cell 29, 380-388, 10.1091/mbc.E17-04-0231.

Shellard, A., and Mayor, R. (2020). All Roads Lead to Directional Cell Migration. Trends Cell Biol. 30 , 852-868, 10.1016/j.tcb.2020.08.002.

Shimizu, T., Yabe, T., Muraoka, O., Yonemura, S., Aramaki, S., Hatta, K., Bae, Y.K., Nojima, H., and Hibi, M. (2005). E-cadherin is required for gastrulation cell movements in zebrafish. Mech. Dev. 122, 747-763.

Smutny, M., Ákos, Z., Grigolon, S., Shamipour, S., Ruprecht, V., Čapek, D., Behrndt, M., Papusheva, E., Tada, M., Hof, B., et al. (2017). Friction forces position the neural anlage. Nat. Cell Biol. 19, 306-317, $10.1038 /$ ncb3492.

Solnica-Krezel, L., Stemple, D.L., and Driever, W. (1995). Transparent things: cell fates and cell movements during early embryogenesis of zebrafish. BioEssays 17, 931-939, 10.1002/bies.950171106. 
Sonavane, P.R., Wang, C., Dzamba, B., Weber, G.F., Periasamy, A., and DeSimone, D.W. (2017). Mechanical and signaling roles for keratin intermediate filaments in the assembly and morphogenesis of mesendoderm tissue at gastrulation. Development dev.155200, 10.1242/dev.155200.

Stachel, S.E., Grunwald, D.J., and Myers, P.Z. (1993). Lithium perturbation and goosecoid expression identify a dorsal specification pathway in the pregastrula zebrafish. Development 117, 1261-1274.

Starruß, J., de Back, W., Brusch, L., and Deutsch, A. (2014). Morpheus: a user-friendly modeling environment for multiscale and multicellular systems biology. Bioinformatics 30, 1331-1332, 10.1093/bioinformatics/btt772.

Swat, M.H., Thomas, G.L., Belmonte, J.M., Shirinifard, A., Hmeljak, D., and Glazier, J.A. (2012). Multiscale modeling of tissues using CompuCell3D. Methods Cell Biol. 110, 325-366, 10.1016/B978-0-12388403-9.00013-8.

Tada, M., and Smith, J.C. (2000). Xwnt11 is a target of Xenopus Brachyury: regulation of gastrulation movements via Dishevelled, but not through the canonical Wnt pathway. Development 127, 22272238.

Tahinci, E., and Symes, K. (2003). Distinct functions of Rho and Rac are required for convergent extension during Xenopus gastrulation. Dev. Biol. 259, 318-335, 10.1016/S0012-1606(03)00206-9.

Thisse, C., Thisse, B., Halpern, M.E., and Postlethwait, J.H. (1994). goosecoid Expression in neurectoderm and mesendoderm is disrupted in zebrafish cyclops gastrulas. Dev. Biol. 164, 420-429, 10.1006/dbio.1994.1212.

Topczewski, J., Sepich, D.S., Myers, D.C., Walker, C., Amores, A., Lele, Z., Hammerschmidt, M., Postlethwait, J., and Solnica-Krezel, L. (2001). The zebrafish glypican knypek controls cell polarity during gastrulation movements of convergent extension. Dev. Cell 1, 251-264, 10.1016/s15345807(01)00005-3.

Twiss, F., Le Duc, Q., Van Der Horst, S., Tabdili, H., Van Der Krogt, G., Wang, N., Rehmann, H., Huveneers, S., Leckband, D.E., and De Rooij, J. (2012). Vinculin-dependent Cadherin mechanosensing regulates efficient epithelial barrier formation. Biol. Open 1, 1128-1140, 10.1242/bio.20122428.

Ulrich, F., Krieg, M., Schotz, E.M., Link, V., Castanon, I., Schnabel, V., Taubenberger, A., Mueller, D., Puech, P.-H.P.-H., Heisenberg, C.-P., et al. (2005). Wnt11 functions in gastrulation by controlling cell cohesion through Rab5c and E-cadherin. Dev. Cell 9, 555-564, 10.1016/j.devcel.2005.08.011.

Vassilev, V., Platek, A., Hiver, S., Enomoto, H., and Takeichi, M. (2017). Catenins Steer Cell Migration via Stabilization of Front-Rear Polarity. Dev. Cell 43, 463-479.e5, 10.1016/j.devcel.2017.10.014.

Vishwakarma, M., Di Russo, J., Probst, D., Schwarz, U.S., Das, T., and Spatz, J.P. (2018). Mechanical interactions among followers determine the emergence of leaders in migrating epithelial cell collectives. Nat. Commun. 9, 3469, 10.1038/s41467-018-05927-6.

Vishwakarma, M., Spatz, J.P., and Das, T. (2020). Mechanobiology of leader-follower dynamics in epithelial cell migration. Curr. Opin. Cell Biol. 66, 97-103, 10.1016/j.ceb.2020.05.007.

Weber, G.F., Bjerke, M.A., and DeSimone, D.W. (2012). A mechanoresponsive cadherin-keratin complex directs polarized protrusive behavior and collective cell migration. Dev. Cell 22, 104-115, 10.1016/j.devcel.2011.10.013.

Wells, S., Nornes, S., and Lardelli, M. (2011). Transgenic zebrafish recapitulating tbx16 gene early developmental expression. PLoS One 6, e21559, 10.1371/journal.pone.0021559.

Xiong, F., Ma, W., Bénazéraf, B., Mahadevan, L., and Pourquié, O. (2020). Mechanical Coupling Coordinates the Co-elongation of Axial and Paraxial Tissues in Avian Embryos. Dev. Cell 55, 354366.e5, 10.1016/j.devcel.2020.08.007. 
Yamada, K.M., and Sixt, M. (2019). Mechanisms of 3D cell migration. Nat. Rev. Mol. Cell Biol. 20, 738752, 10.1038/s41580-019-0172-9.

Yamashita, S., Miyagi, C., Carmany-Rampey, A., Shimizu, T., Fujii, R., Schier, A.F., and Hirano, T. (2002). Stat3 Controls Cell Movements during Zebrafish Gastrulation. Dev. Cell 2, 363-375.

Yamashita, S., Miyagi, C., Fukada, T., Kagara, N., Che, Y.-S., and Hirano, T. (2004). Zinc transporter LIVI controls epithelial-mesenchymal transition in zebrafish gastrula organizer. Nature 429, 298-302, 10.1038/nature02545.

Yonemura, S., Wada, Y., Watanabe, T., Nagafuchi, A., and Shibata, M. (2010). alpha-Catenin as a tension transducer that induces adherens junction development. Nat. Cell Biol. 12, 533-542, 10.1038/ncb2055. 


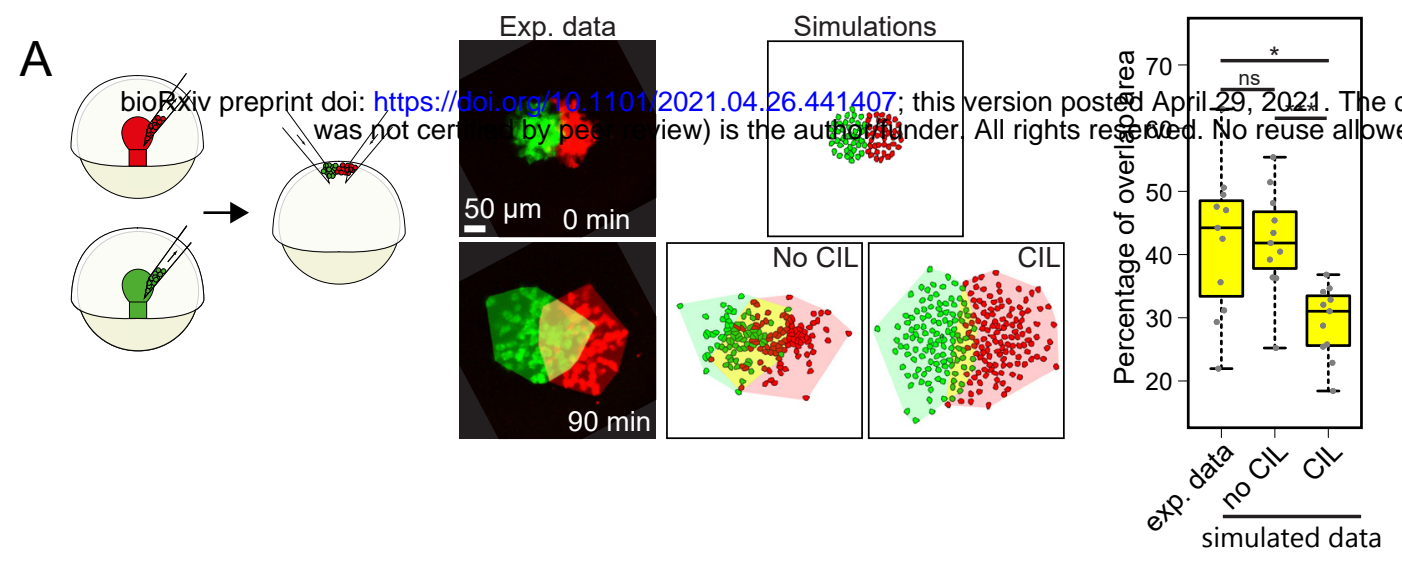

B
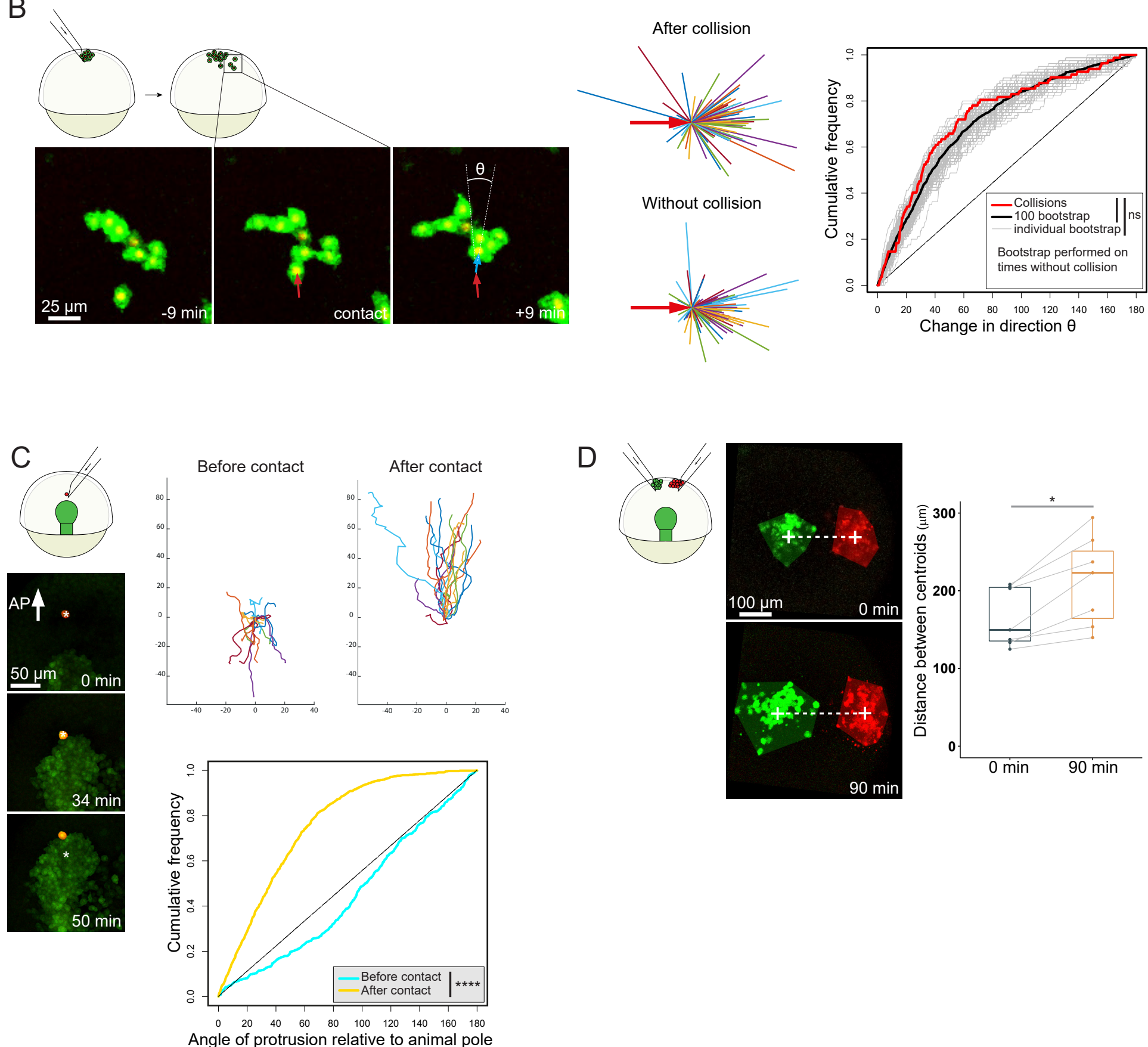

Figure 1 
A
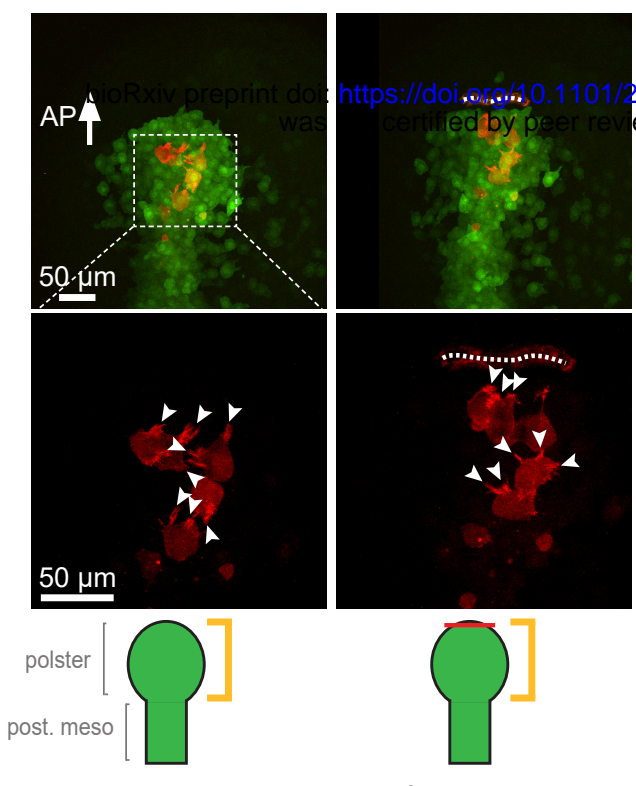

B

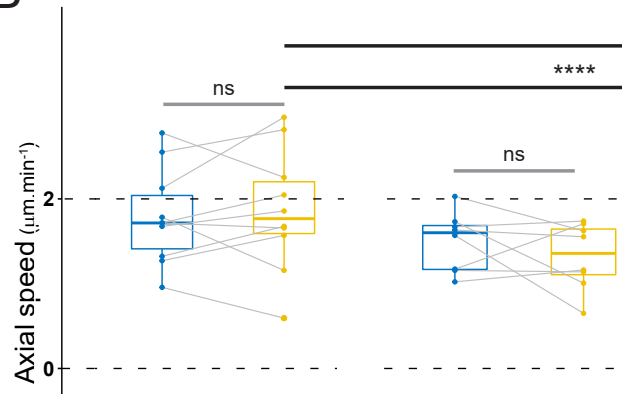

$0-10 \min 11-34 \mathrm{~min}$ before after ablation
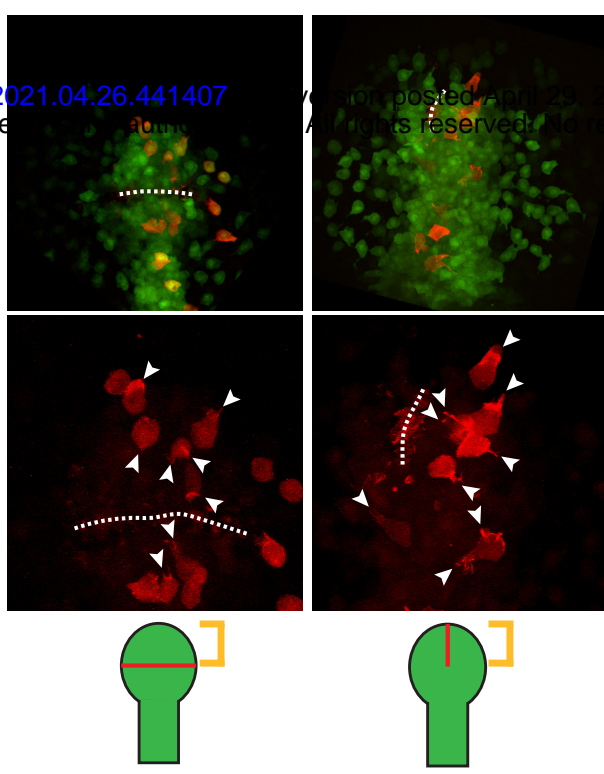

ablation at mid-polster
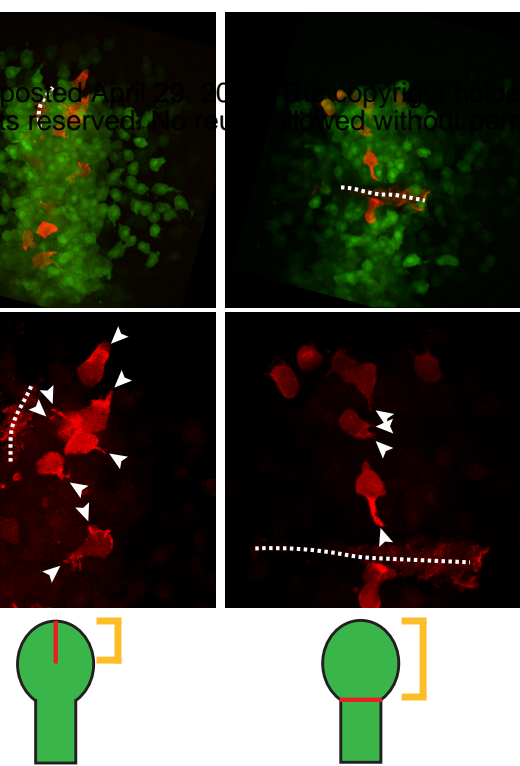

control ablation

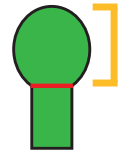

ablation between polste and post. mesoderm
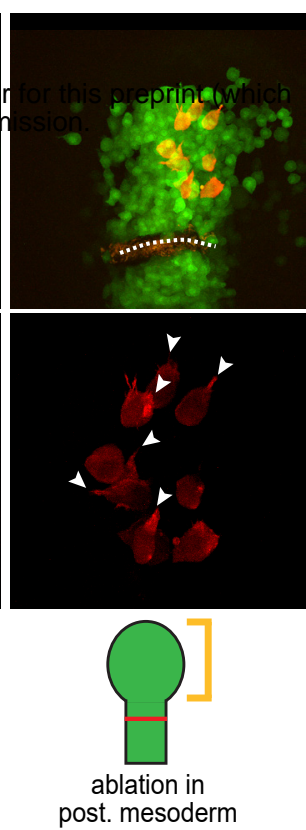

post. mesoderm

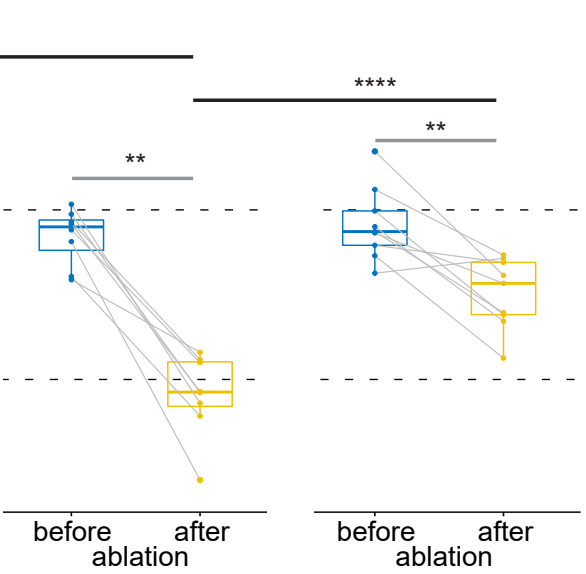

C
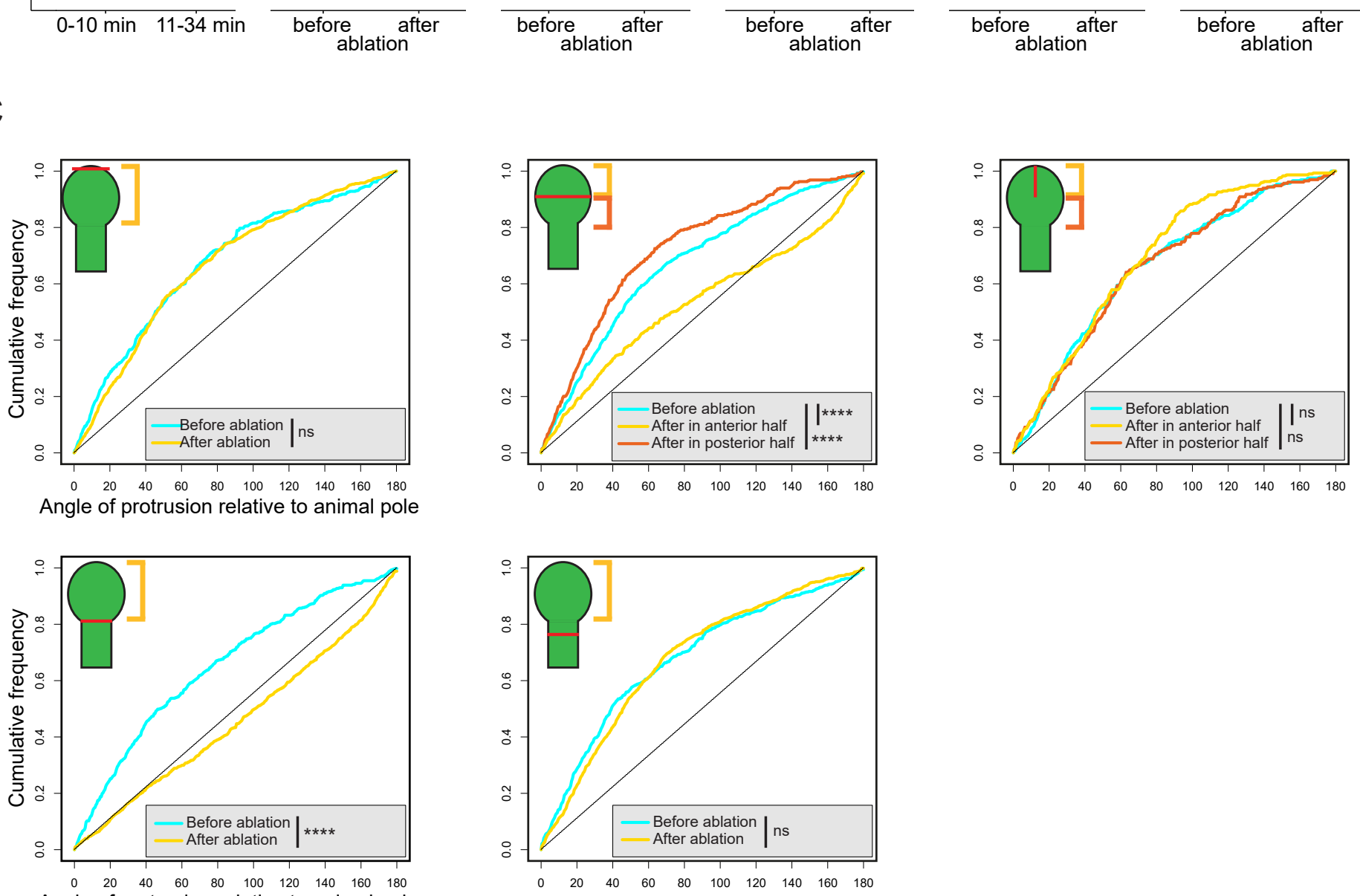

Angle of protrusion relative to animal pole

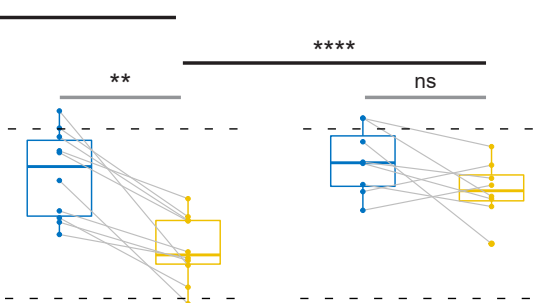

Figure 2 


\section{A \\ WT \\ Removed polster}

bioRxiv preprint doi: https://doi.org/10.1101/2021.04.26.441407; this version posted April was not certified by peer review) is the author/funder. All rights reserved.
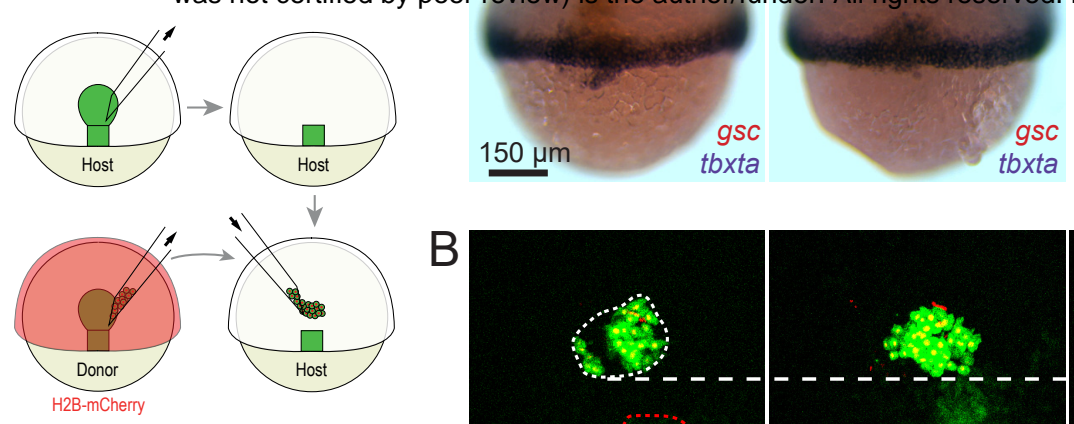

$50 \mu \mathrm{m}$

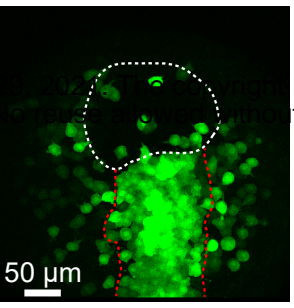

holder for this preprint (which permission.

$B$
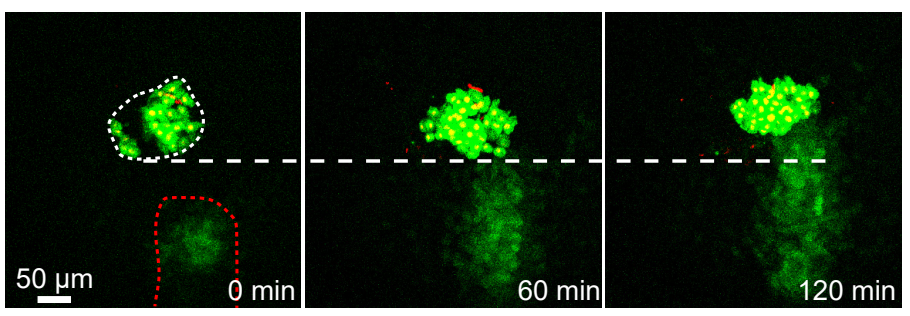

C

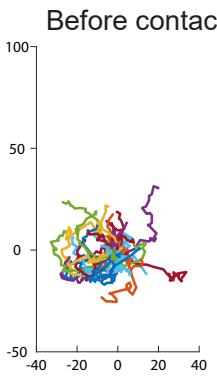

After contact

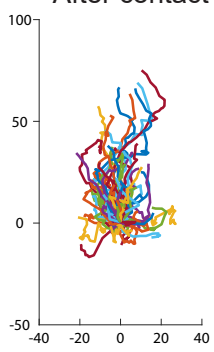

D

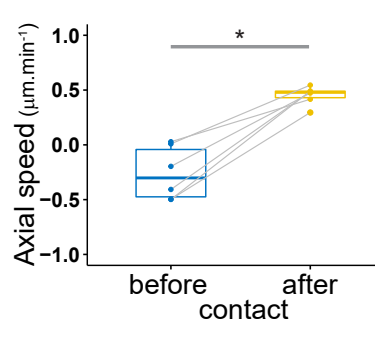

E

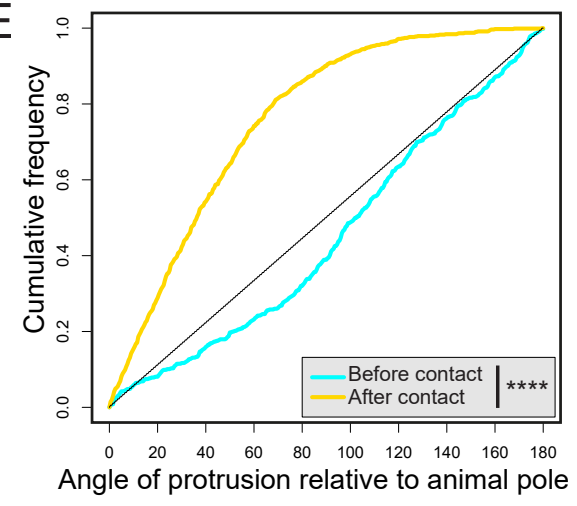

Figure 3 

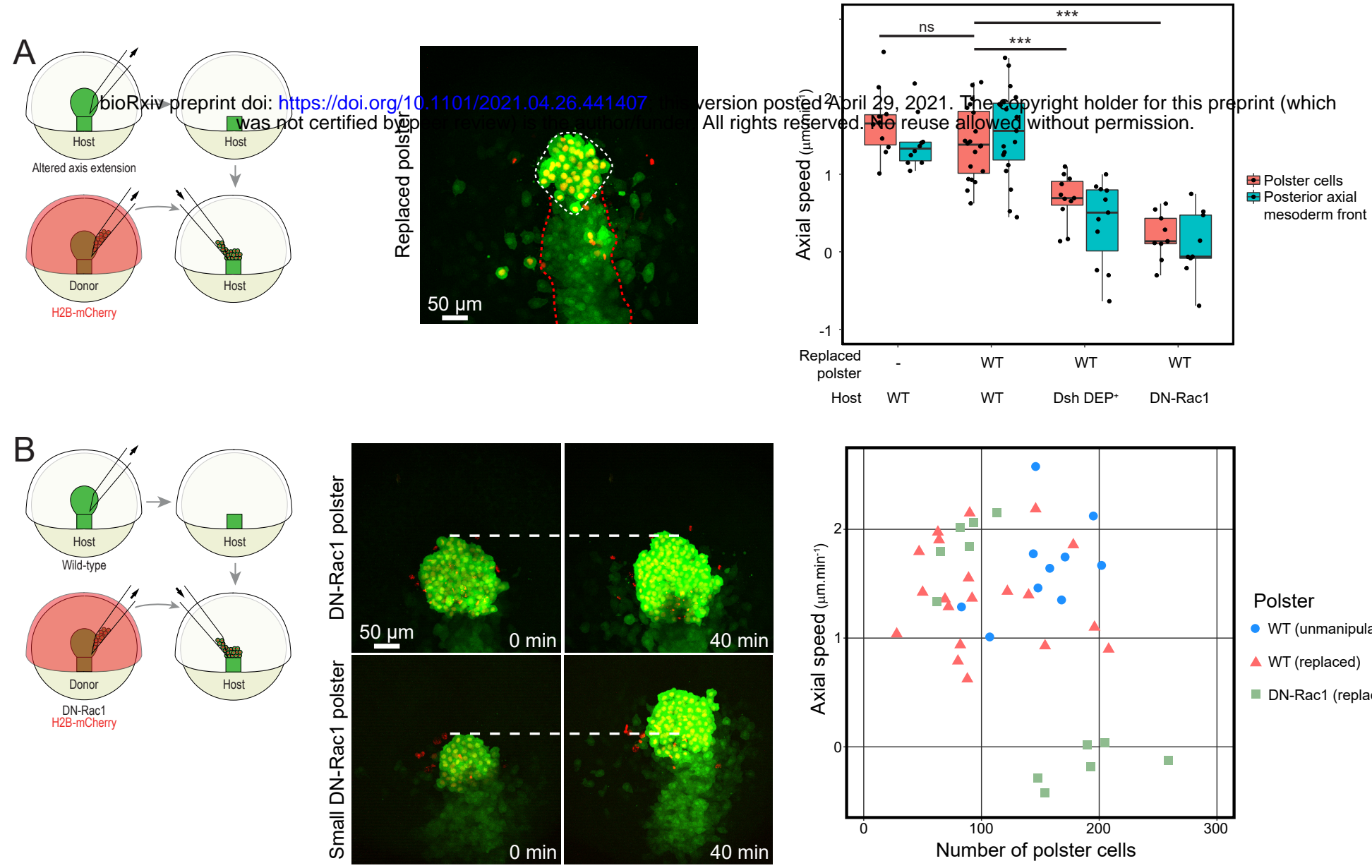

Polster cells

version postêt 2 A pril 29 , 2021. The ofpyright holder for this preprint (which All rights reserv d. reuse allowed without permission.

C
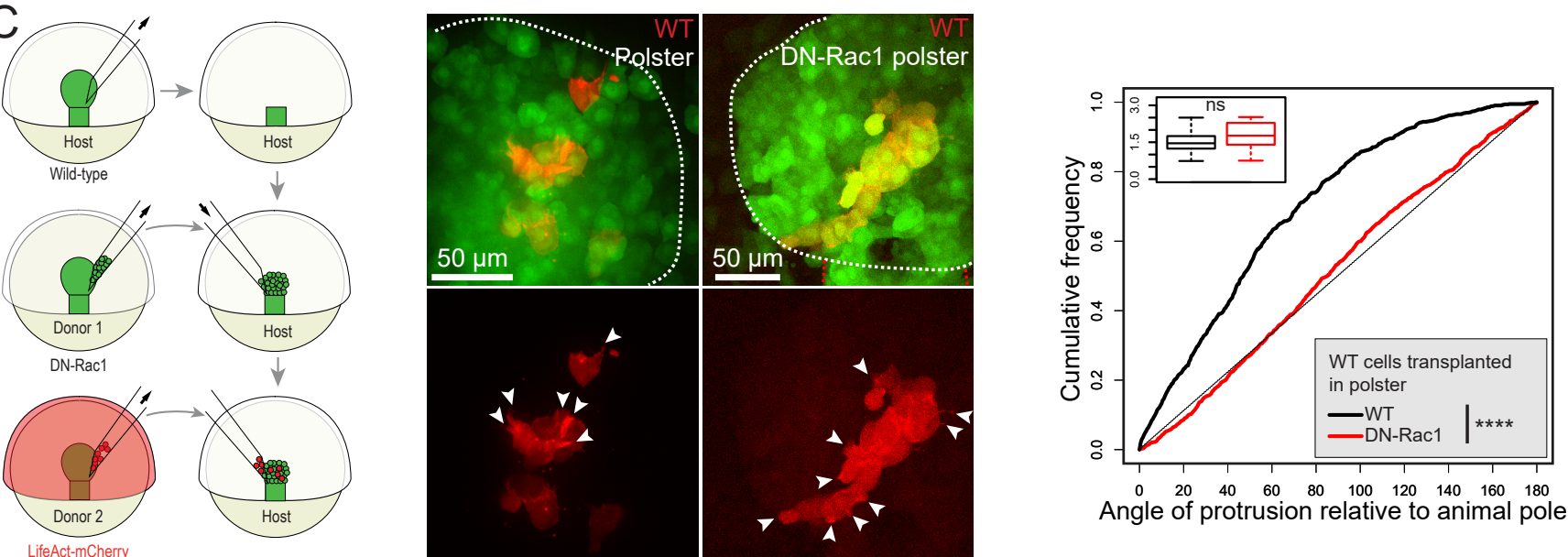

Polster

WT (unmanipulated)

A WT (replaced)

DN-Rac1 (replaced)

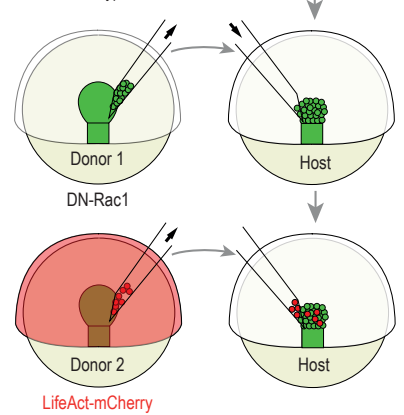

$\mathrm{D}$
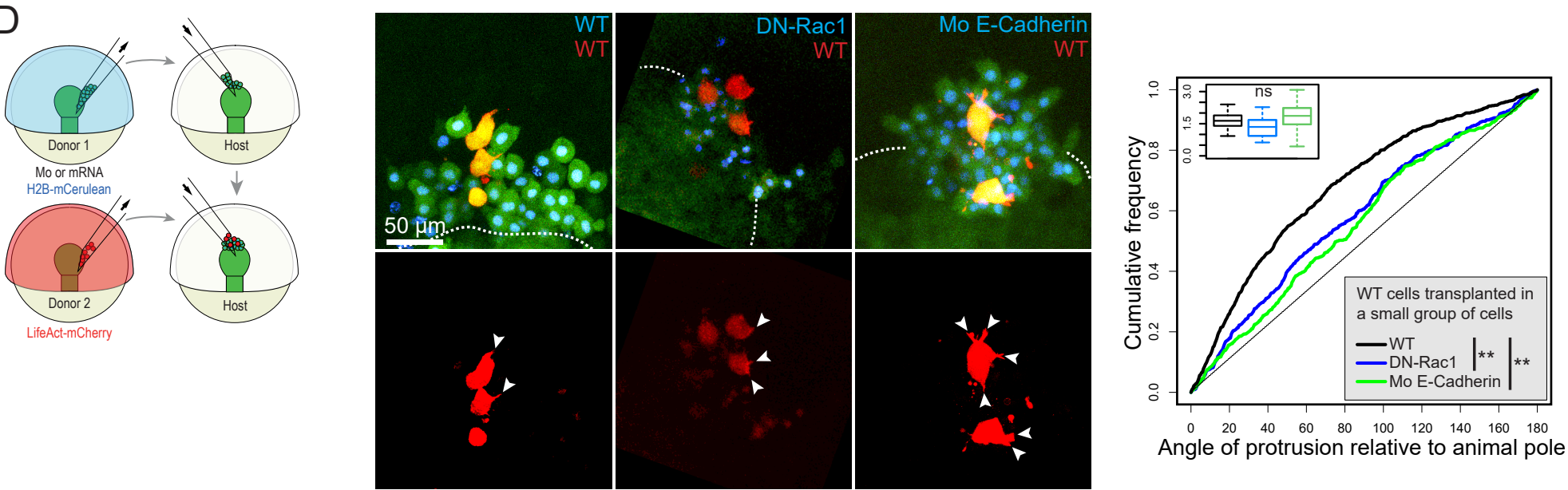

Figure 4 


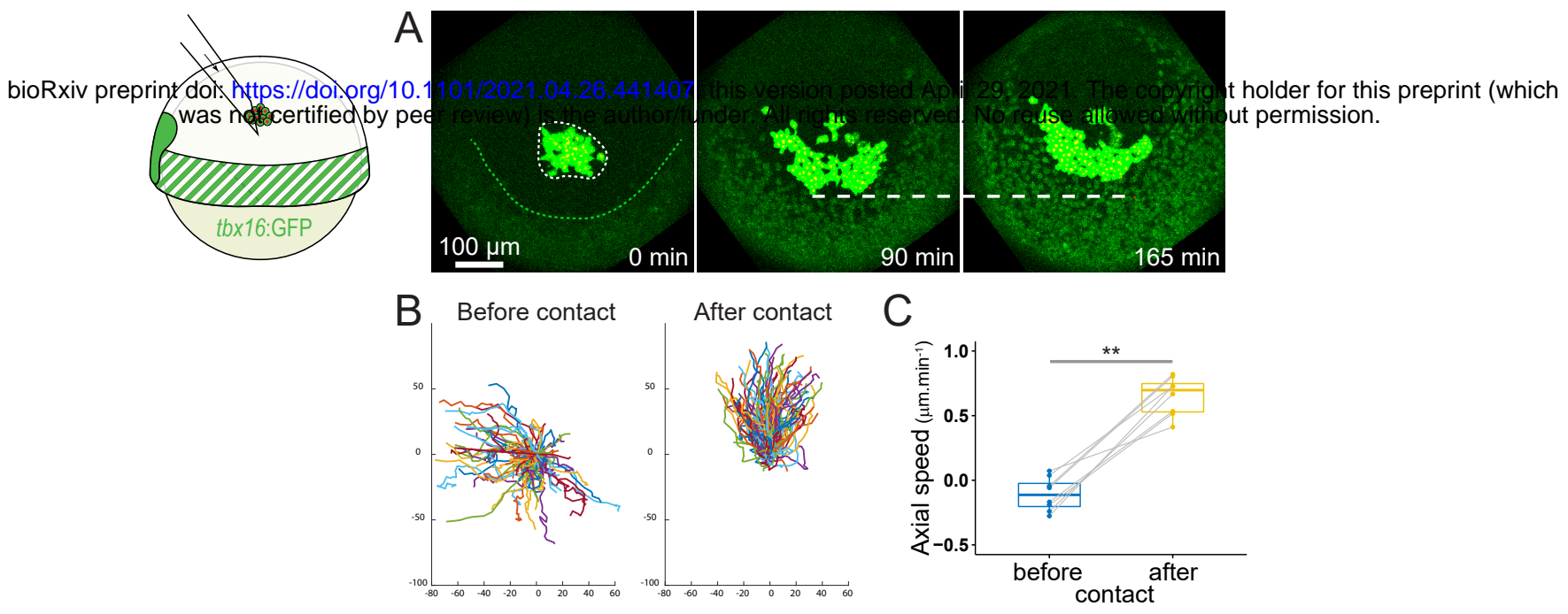

D
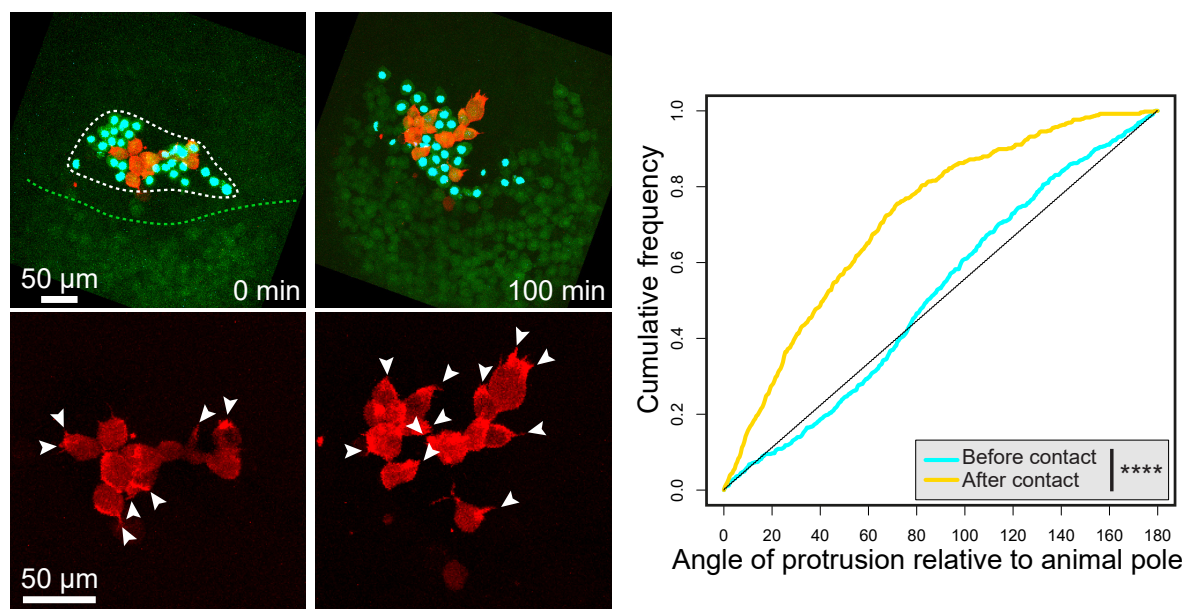

Angle of protrusion relative to animal pole

Figure 5 


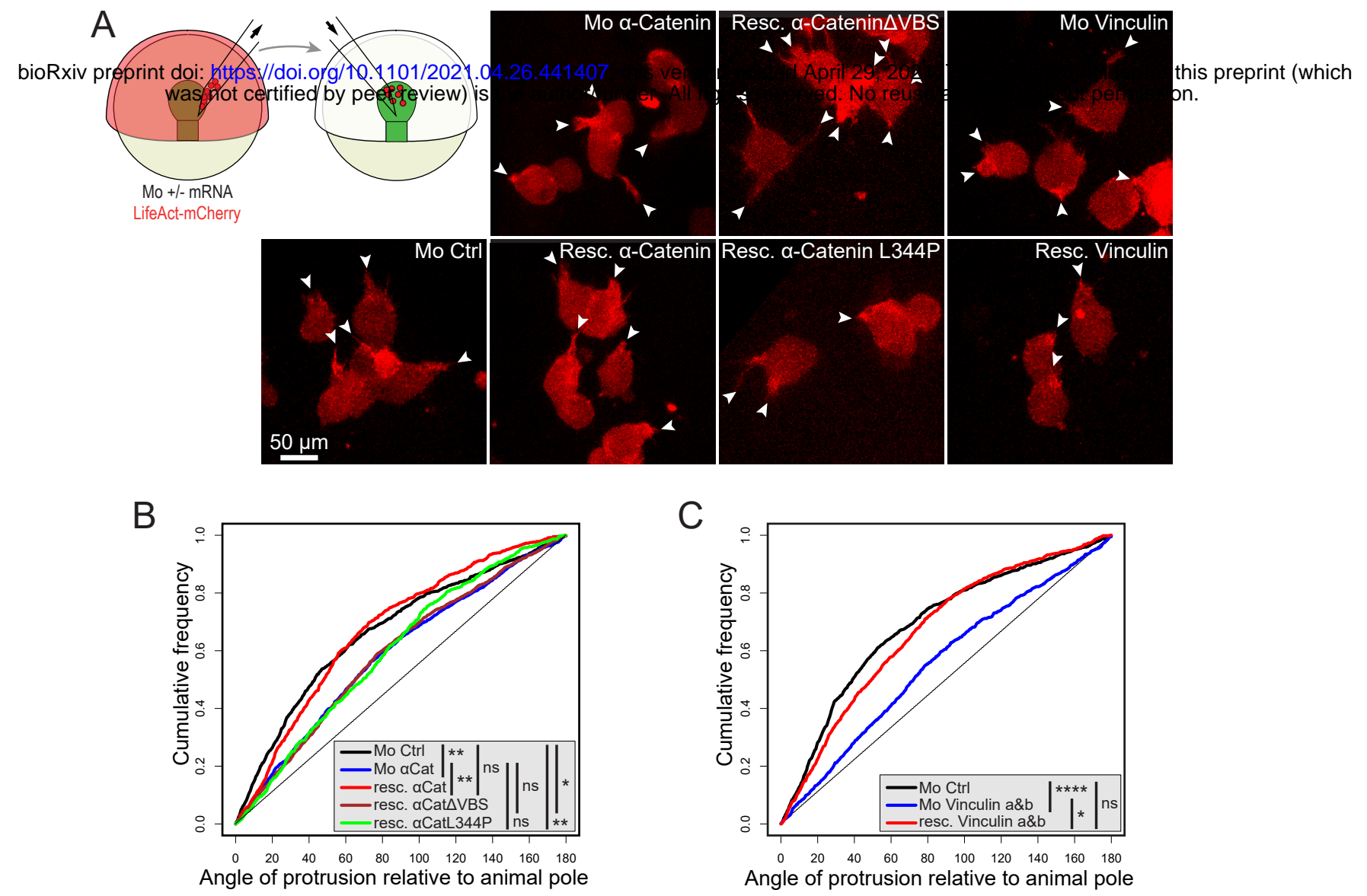

Figure 6 


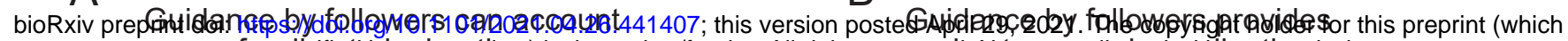

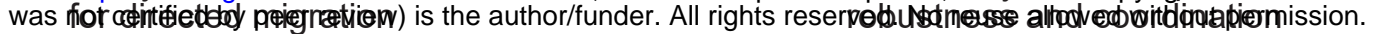
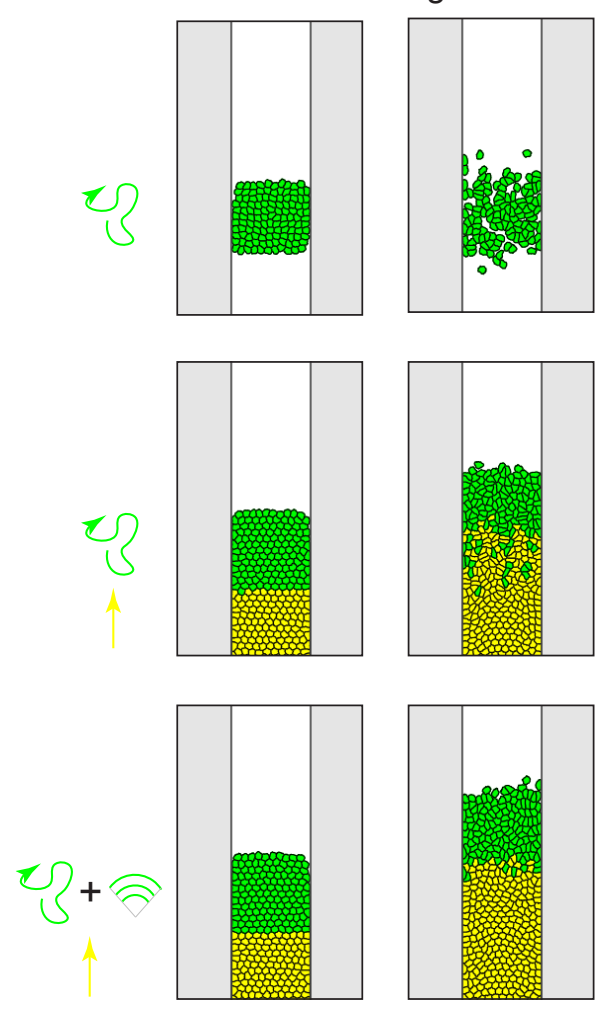

$\int$ Run and Tumble

C

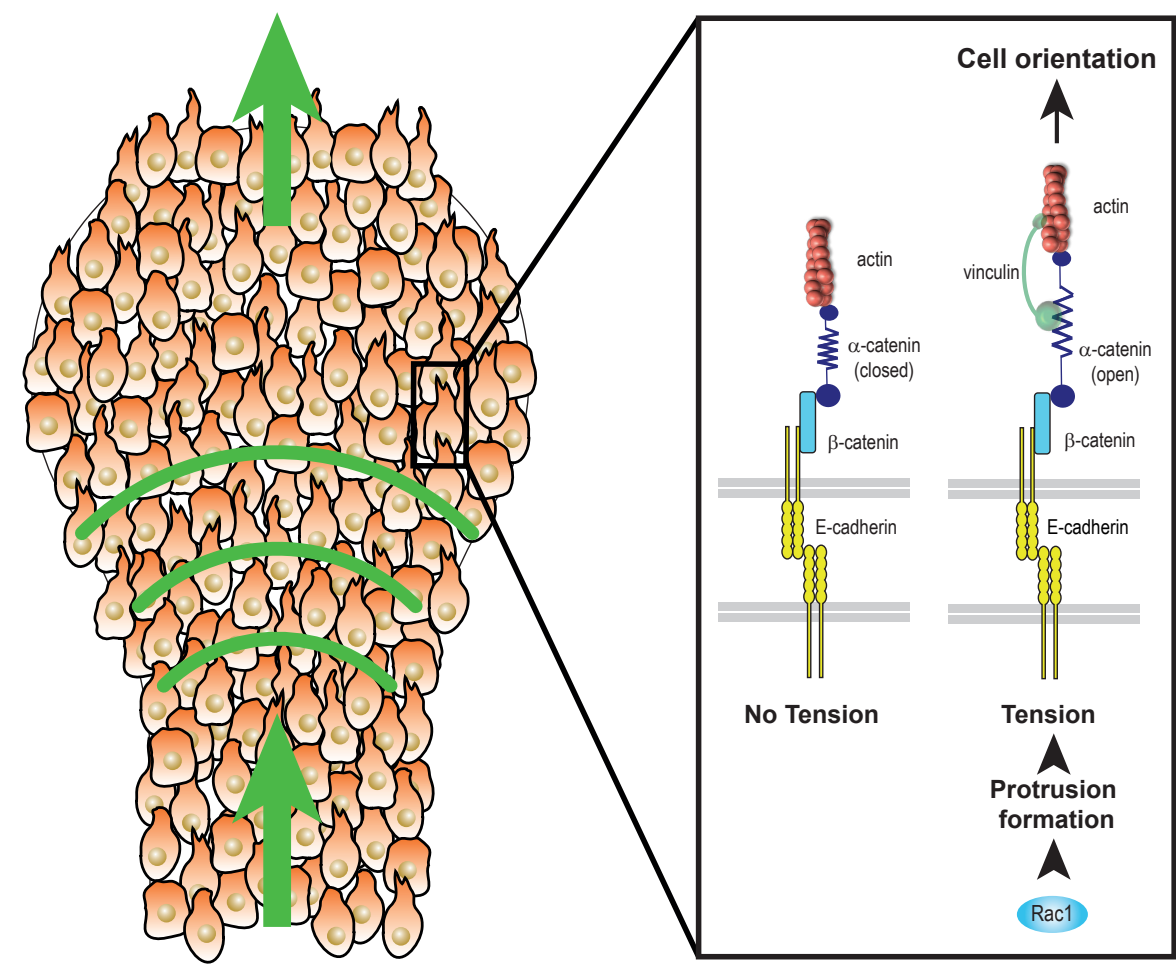

Figure 7
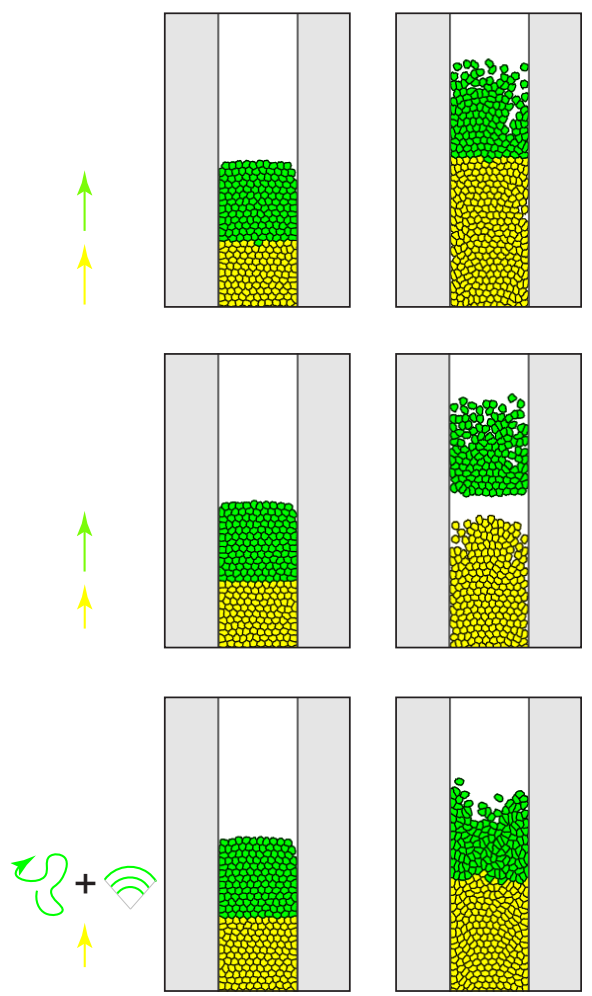
by followers 